\title{
FLUCTUATIONS AND SIMPLE CHAOTIC DYNAMICS
}

\author{
J.P. CRUTCHFIELD and J.D. FARMER \\ Physics Board of Studies, University of California, Santa Cruz, California 95064, U.S.A. \\ and
}

\section{B.A. HUBERMAN}

Xerox Palo Alto Research Center, Palo Alto, California 94304, U.S.A.

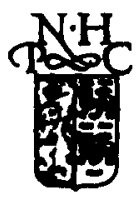

NORTH-HOLLAND PUBLISHING COMPANY - AMSTERDAM 


\title{
FLUCTUATIONS AND SIMPLE CHAOTIC DYNAMICS
}

\author{
J.P. CRUTCHFIELD and J.D. FARMER* \\ Physics Board of Studies, University of California, Santa Cruz, California 95064, U.S.A.
}

and

\section{B.A. HUBERMAN}

Xerox Palo Alto Research Center, Palo Alto, California 94304, U.S.A.

Received July 1982

Contents:

1. Introduction

2. Dynamics in the absence of fluctuations

3. Dynamics in the presence of fluctuations

4. Noise as a disordering field

5. Concluding remarks
47

48

57

68

74
Appendix A. Fluctuations in a driven anharmonic oscillator

Appendix B. Characteristic exponents in period-doubling regimes

Appendix C. Equivalence of parametric and additive noise References

\begin{abstract}
:
We describe the effects of fluctuations on the period-doubling bifurcation to chaos. We study the dynamics of maps of the interval in the absence of noise and numerically verify the scaling behavior of the Lyapunov characteristic exponent near the transition to chaos. As previously shown, fluctuations produce a gap in the period-doubling bifurcation sequence. We show that this implies a scaling behavior for the chaotic threshold and determine the associated critical exponent. By considering fluctuations as a disordering field on the deterministic dynamics, we obtain scaling relations between various critical exponents relating the effect of noise on the Lyapunov characteristic exponent. A rule is developed to explain the effects of additive noise at fixed parameter value from the deterministic dynamics at nearby parameter values.
\end{abstract}

* Present address: Center for Nonlinear Studies, MS B 258, Los Alamos Laboratories, Los Alamos, New Mexico 87545, U.S.A.

Single orders for this issue

PHYSICS REPORTS (Review Section of Physics Letters) 92, No. 2 (1982) 45-82.

Copies of this issue may be obtained at the price given below. All orders should be sent directly to the Publisher. Orders must be accompanied by check.

Single issue price Dfl. 20.00 , postage included. 


\section{Introduction}

Chaotic dynamical systems provide a significant new addition to the conventional dynamical repertoire of equilibrium and periodic oscillation. Central to their usefulness in describing observed random behavior is the issue of their stability and the observability of their bifurcation sequences in the presence of noise sources. In particular, given the fact that fluctuations are an important aspect of many-body systems, one would like to understand the role of fluctuations vis á vis the chaotic behavior generated by deterministic nonlinear dynamics.

Recently we have shown [1] that for a certain class of processes the effect of external fluctuations on the onset and properties of chaos can be described in fairly simple fashion. For systems displaying period-doubling bifurcations we found that the presence of noise leads to both a gap in the bifurcation sequence and a renormalization of the chaotic threshold. Furthermore, we noticed that for fixed parameter values the addition of noise to a nonlinear deterministic equation produces additional bifurcations, $\dagger$ which can be observed in measurable properties such as power spectra. These effects are of importance to current attempts at understanding turbulent behavior of fluids and solids in terms of chaotic, deterministic models.

In this paper we discuss in detail the effects of fluctuations on the cascade bifurcation to chaos. By the cascade bifurcation we shall mean not only the infinite sequence of subharmonic bifurcations, at each stage of which the period of a limit cycle is doubled, but also the symmetric bifurcation sequence above the chaotic threshold, in which $n$ bands of a chaotic attractor merge pairwise to form an $n / 2$-band attractor. Ample illustration of the cascade bifurcation follows in the next section.

Previous work discussed the effect of external fluctuations on the cascade bifurcation found in a driven nonlinear oscillator [1]. That study was motivated in part by the fact that in condensed matter systems thermal fluctuations play an important role which had to be incorporated into the nonlinear equations leading to solid-state turbulence [2]. More generally, though, the cascade bifurcation sequence and its alteration in the presence of fluctuations is of interest in systems that range from fluid flows $[3,4]$ to noise phenomena in solid-state systems [5]. Moreover, this bifurcation sequence is found in numerical studies of a wide range of mathematical models, including nonlinear ordinary $[6,7]$ and partial [8] differential equations. In the experimental observation of bifurcation sequences in Bénard flow [3] and spherical Couette flow [4] one observes only a finite number $(<4)$ of bifurcations in the cascade sequence, whereas the scaling theory developed by Feigenbaum [9] and Collet and Eckmann [10] for the period-doubling half of the cascade requires that the dynamics undergo an infinite number of bifurcations before the transition to chaos. Indeed, it is in just this limit of infinite bifurcation that the scaling theory becomes exact. As previously suggested [1], this discrepancy can be explained by the interaction of external fluctuations and the deterministic sequence of bifurcations.

From a study of the geometry of the attractors found in the driven anharmonic oscillator [1], we found that increased noise levels could induce a transition to chaotic behavior. Furthermore, rather than destabilizing or erradicating chaotic motions in the phase space, noise enhanced the chaotic behavior, while destroying periodic orbits. That is, the local instabilities responsible for the deterministic chaotic behavior actually increased the observability of chaos in the presence of fluctuations. Using Feigenbaum's scaling theory and the existence of the bifurcation gap, we derived a scaling relationship for the noise dependence of observable bifurcations in a cascade. These features were also found in a one-dimensional map typical of those for which the universal scaling theory was developed [1].

The present paper reports in more detail the results of our study of one-dimensional maps, that is, of

\footnotetext{
† We employ a broader definition of bifurcation than is typical: an observable, qualitative change in a system's behavior as a control parameter is slowly varied.
} 
nonlinear transformations of the unit interval onto itself. The main results of our paper can be summarized as follows. In the absence of noise, we verify numerically the scaling predictions of Huberman and Rudnick for the behavior of the Lyapunov characteristic exponent $\lambda$ and discuss several other features of $\lambda$ found in the cascade bifurcation. The Lyapunov characteristic exponent can be thought of as a disorder parameter for chaos [11]. We show that the existence of a bifurcation gap implies a scaling behavior for the chaotic threshold and determine the associated exponent. By realizing that fluctuations act as a disordering field on the deterministic dynamics, we obtain a scaling relation between various critical exponents relating the effect of noise on $\lambda$, and develop the notion of a noise susceptibility. We also show that even in the periodic regime there is a non-trivial effect of external fluctuations on the period-doubling bifurcations. This is reflected in the fact that not only higher periods become obliterated by increasing noise, but the bifurcation points themselves become blurred. In particular, at the points of bifurcation, $\lambda$ no longer vanishes as it does in the deterministic limit.

In section 2 we review the dynamics of maps of the interval in the absence of noise. We present a typical map which displays the cascade bifurcation and analyze it in detail. We summarize the scaling theory of bifurcations and discuss the Lyapunov characteristic exponent as a measure of the stability of the asymptotic behavior. Section 3 considers the effects of noise on the cascade bifurcation sequence and the onset of chaos. A rule is developed that allows the effect of noise at a fixed parameter value to be predicted from the knowledge of the purely deterministic dynamics at adjacent parameter values. In section 4 we discuss the role of noise as a disordering field and the scaling behavior of the noise critical exponents. Section 5 contains a summary of the scaling ideas and discusses the applicability of these ideas to physical systems. We then mention other questions related to the interaction of chaotic dynamics, external fluctuations, and observational noise. A set of appendices discusses details of the effect of fluctuations on a driven anharmonic oscillator, the scaling of the characteristic exponent within the period-doubling regime, and the noise equivalence rule of section 3 .

\section{Dynamics in the absence of fluctuations}

Dynamical systems theory [12] describes the time evolution of a system as a trajectory, or an orbit, in a phase space of the system's possible states. Typically, the physically interesting behavior of a system is that which is observed after initial transients have died away. The set of states which an orbit eventually visits is called the system's attractor. The study of dynamical systems considers not only the structure of attractors but also the qualitative change, or bifurcation, from one type to another as some parameter is smoothly varied.

Since the first physically-motivated study of chaotic dynamics by Lorenz [13], one-dimensional (1D) maps have played a fundamental role in the field's development despite their apparent simplicity. The ID map obtained from a system of ordinary differential equations captures the essential geometry underlying the chaotic dynamics. Although such a reduction of dimension (from three to one, in Lorenz's case) cannot be uniformly applied to all dynamical systems, for many problems the technique provides more than sufficient heuristic insight into the processes responsible for chaotic behavior. Specifically, by identifying all the points which asymptotically merge, that is, all the points on the same stable manifold, it is possible to summarize many of the properties of a simple chaotic attractor in three dimensions by a 1D map.

For dissipative systems with a chaotic attractor that appears locally two-dimensional, a cross section through the attractor intersects it in some curve. One can then consider the dynamics as a map from this cross section onto itself; this map is called the Poincare map. By parametrizing points on the curve of 
intersection (from 0 to 1 ) and collecting a set of successive points $\left\{x_{1}, x_{2}, x_{3}, \ldots\right\}$ as an orbit passes through the section, the dynamics of the attractor can be summarized in a one-dimensional map of the form

$$
x_{n+1}=f\left(x_{n}\right), \quad n=1,2,3, \ldots,
$$

where $x_{n}$ is the $n$th crossing of the orbit through the section and where $f$ is a nonlinear function on the unit interval. In this sense, discrete time maps summarize the dynamics underlying chaotic behavior found in higher dimensional systems. From their simplicity, 1D maps have developed as prototypical models in the study of chaotic dynamics [14].

For dissipative dynamical systems, such as discrete mappings and ordinary and partial differential equations, that exhibit cascading bifurcations, the dynamics can be described in practice by a 1D map with a single smooth maximum. An example of such maps is provided by the logistic equation, which is defined by

$$
x_{n+1}=r x_{n}\left(1-x_{n}\right), \quad 0<x_{n}<1,
$$

and where the bifurcation parameter $r(0<r<4)$ determines the height of the quadratic function $f(x)=x(1-x)$. As a graphic example of the complexity present in this class of maps, the bifurcation diagram of fig. 1 presents the change in the attractor of eq. (2-2) as a function of the bifurcation parameter $r$ in the regime $[3,4]$.

At a fixed parameter value in the bifurcation diagram, a periodic orbit consists of a countable set of points, while a chaotic attractor fills out dense bands within the unit interval. Figure 2 shows the probability density for the two bands at $r=3.59687$. The dominant bifurcation sequence seen in fig. 1 is the single $2^{n}$ cascade, through which the attractor first becomes chaotic and eventually fills the interval via the pairwise merging of bands. The period-doubling and band merging accumulates at a value

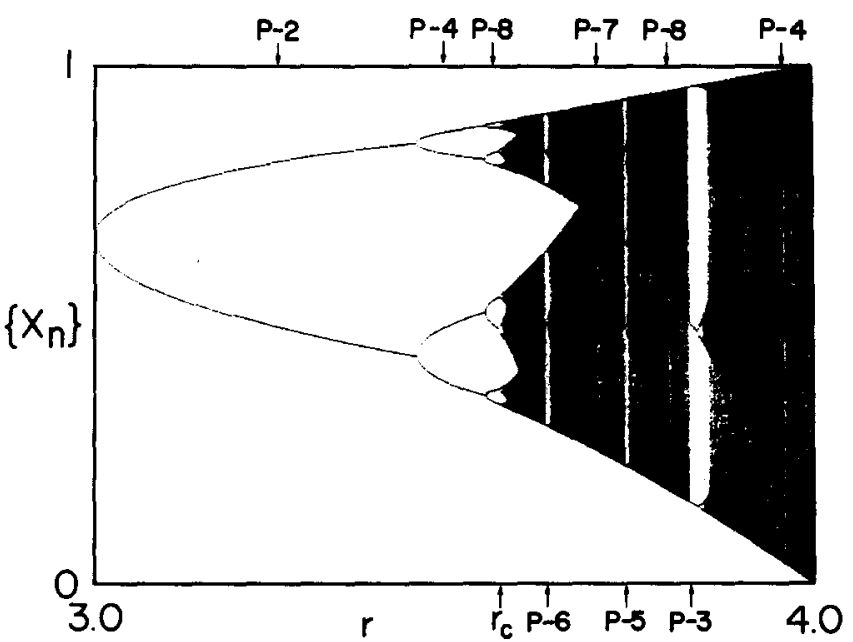

Fig. 1. The attractor versus bifurcation parameter $r$ for the logistic map, eq. (2-2), $x_{n+1}=r x_{n}\left(1-x_{n}\right) .700$ iterations plotted after an initial 500 iterations for each increment in the bifurcation parameter. The parameter was incremented 1000 times in the interval $[3,4]$. For the sake of clarity and resolution, only the bifurcation diagram for $r$ in $[3,4]$ is shown. For $r$ in $[0,1], x_{n}=0$ is the stable behavior; and for $r$ in $[1,3]$, one has a stable fixed point described by $x=(r-1) / r$. 


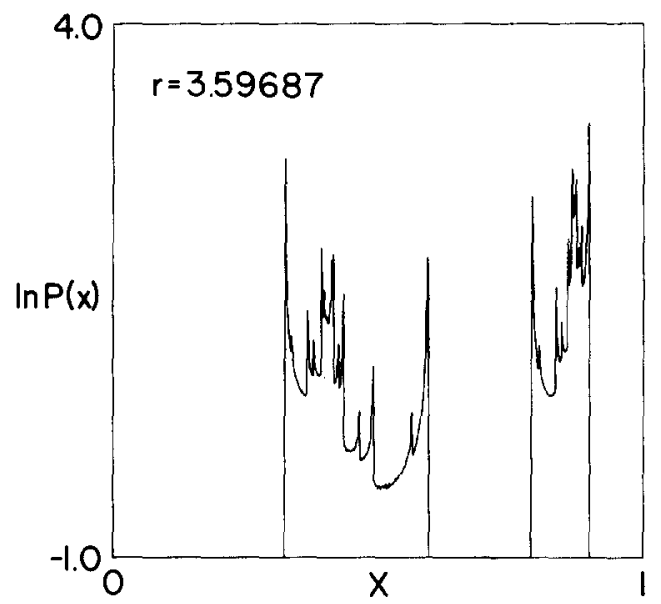

Fig. 2. Normalized probability density $P(x)$ of the two band attractor at $r=3.59687$ shown on a logarithmic scale. $P(x)$ is a histogram of $10^{7}$ iterations of eq. (2-2) partitioned into 1000 bins. The peaks in $P(x)$ are successive images of the maximum, or critical point, $x_{\mathfrak{c}}$. Note that each band is a mirror image of the other.

$r_{\mathrm{c}}=3.569945672 \ldots$ after an infinite number of bifurcations. In the chaotic regime above $r_{\mathrm{c}}$, one finds small windows of higher period cascades with periods $q \times 2^{n}$, with $q$ an integer and where $n$ denotes the degree of the period-doubling of a fundamental periodic orbit of period $q$. Within each such window, one also finds the associated reverse bifurcation [7] of $q \times 2^{n+1}$ bands merging into $q \times 2^{n}$ bands. In what follows we will call $q$ the periodicity of the cascade; $q=1$ for the primary cascade described above [15].

To describe in more detail the structure apparent in fig. 1 we now focus on the successive images of the map's maximum, called the map's critical point $x_{c}$, where the slope vanishes. One of the more striking features of the bifurcation diagram above $r_{c}$ is the veil-like structure highlighted by dark lines which vary smoothly with the parameter. As the attractors in the chaotic regime consist of dense subsets of the interval rather than discrete points, one needs to consider the action of the map on a probability distribution. The dark lines in the diagram correspond, then, to successive images of the critical point and indicate regions of high probability density. These are seen in fig. 2 as spikes in the probability density. To describe their dependence on the bifurcation parameter $r$, it is useful to write

$$
x_{n+1}=F\left(r, x_{n}\right) \text {, }
$$

with $F(r, x)=r x(1-x)$ in our example. Then the $m$ th image of the critical point, $x_{\mathrm{c}}=0.5$, is a polynomial in $r, F^{m}(r, 0.5)$, where $F^{m}(r, x)=F\left(F^{m-1}(r, x)\right)$. For example, above $r_{\mathrm{c}}$, the first image of $x_{\mathrm{c}}$ defines the upper bound on $\left\{x_{n}\right\}$; it is the straight line

$$
F\left(r, x_{\mathrm{c}}\right)=r / 4 \text {, }
$$

seen in fig. 1. Similarly, the second image defines the lower bound on $\left\{x_{n}\right\}$ which is given by

$$
F^{2}\left(r, x_{\mathrm{c}}\right)=\frac{r^{2}}{4}\left(1-\frac{r}{4}\right)
$$

Further iterates of the critical point must lie between these two. For example, the third iterate 


$$
F^{3}\left(r, x_{c}\right)=\frac{r^{3}}{4}\left(1-\frac{r}{4}\right)\left(1-\frac{r^{2}}{4}\left(1-\frac{r}{4}\right)\right)
$$

is a lower bound on the upper band in the two-band region. Similarly, the fourth iterate $F^{4}\left(r, x_{c}\right)$ is an upper bound on the lower band in the two band region. The pair of bands merges into one band at a parameter value $r_{\text {merge }}$ determined by the intersection of $F^{3}\left(r, x_{\mathrm{c}}\right)$ and $F^{4}\left(r, x_{\mathrm{c}}\right)$, that is, where

$$
F^{3}\left(r, x_{\mathrm{c}}\right)=F^{4}\left(r, x_{\mathrm{c}}\right)=F\left(r, F^{3}\left(r, x_{\mathrm{c}}\right)\right)
$$

or

$$
1=r\left(1-F^{3}\left(r, x_{\mathrm{c}}\right)\right)
$$

which gives $r_{\text {merge }}=3.67857351 \ldots$. It is now apparent that each of the dark lines in the chaotic region of the bifurcation diagram corresponds to one of the images of the critical point $x_{c}$.

The appearance of stable periodic orbits within the chaotic regime is signalled by the qth iterate of the critical point mapping onto itself; that is,

$$
F^{m+q}\left(r, x_{\mathrm{c}}\right)=F^{m}\left(r, x_{\mathrm{c}}\right), \quad m=1,2,3, \ldots
$$

As can be seen in the diagram, the labeled $q$-periodic regimes coincide with the crossing of the $q$ th iterate of the critical point $F^{q}\left(r, x_{\mathrm{c}}\right)$ through $x_{\mathrm{c}}=0.5$. In fact, the period $q$ orbit becomes stable just before this point which is the point of superstability. The number of windows of a particular periodicity increases with $q$ and each of these windows is generally of different width. Furthermore, the width of these windows decreases rapidly with increasing $q$ [7].

In the chaotic regime, one can see the unstable remnants of the orbits of period $2^{n}$ which were stable below $r_{\mathrm{c}}$. Indeed, once an orbit comes into existence at a given parameter value it does not vanish at higher parameter values, although its stability may change. These remnants are apparent as particularly low values in the probability density within the chaotic bands and emanate from the points at which the bands join. The white streaks corresponding to these unstable orbits are seen most readily when viewing fig. 1 from the side, looking parallel to the $r$-axis in the direction of decreasing $r$.

Despite the existence of such detailed structure, the bifurcation diagram exhibits a high degree of self-similarity. By self-similarity we mean the property of objects whose structure, as observed on one length scale, is repeated on successively smaller scales [16]. To describe this, we denote the value of $r$ at a bifurcation by $r_{m-n}$, where, if $m<n$, a periodic orbit bifurcates from period $m$ to period $n$ and, if $m>n, m$ bands merge into $n$ bands. If we consider the bifurcation diagram for $r$ in $[1,4]$ as the first scale, where the attractor begins just above $r=1$ as a period 1 orbit and ends at $r=4.0$ as a single chaotic band, then this structure is repeated twice on a reduced scale within the parameter subinterval. $\left[r_{1-2}, r_{2-1}\right]=[3,3.67857 \ldots]$, four times in the still smaller regime $\left[r_{2-4}, r_{4-2}\right]=[3.44944 \ldots, 3.59257 \ldots]$ and so on. In this manner, the period 6 regime can be thought of as two copies of the period 3 regime. Thus one need only describe in detail the periodic regimes in $\left[r_{2-1}, 4\right]=[3.67857 \ldots, 4]$ in order to understand the periodic regimes in $\left[r_{\mathrm{c}}, r_{2-1}\right]=[3.56995 \ldots, 3.67857 \ldots]$. Within this scheme, the period 7 window just below $r=4.0$ gives rise to a period 14 counterpart just below $r_{2-1}$. Such self-similarity is also found within each window of higher periodicity.

Feigenbaum [9] has developed a scaling theory for the non-chaotic period-doubling side of the cascade bifurcation in $1 \mathrm{D}$ maps which exactly describes this self-similarity in the limit of highly 
bifurcated attractors. This theory predicts that the parameters $r_{n}$ at the bifurcation from a period $2^{n}$ to a period $2^{n+1}$ orbit scale according to

$$
\left(r_{\mathrm{c}}-r_{n}\right) \sim \delta^{-n}
$$

where $\delta=4.69920 \ldots$ depends only on the (quadratic) nature of the maximum of the map. It also predicts that the width of bifurcation "forks" in the periodic regime which stradle $x_{\mathrm{c}}$ decreases by a spatial rescaling constant $\alpha=2.502907876 \ldots$ Lorenz [7], in turn, has shown that the bifurcation parameters for the band joinings in the chaotic regime also scale in this manner and that the width of the band containing $x_{\mathrm{c}}$ scales by $\alpha$ at band-merging bifurcations. Grossman and Thomae were apparently the first to measure the scaling factor $\delta$ for both the period-doubling and band-merging bifurcations [15]. Furthermore, the bifurcation parameters describing the cascades in all of the windows of higher periodicity exhibit this scaling behavior.

In the study of chaotic dynamics it is often useful to have some measure of the degree of randomness generated by the deterministic equations. Several quantities have been developed for this, but we shall only consider those related to orbital stability. Orbital stability for a given orbit depends on the behavior of its neighboring orbits. If points near an orbit converge toward it, then it is stable to small perturbations and is said to be locally stable. An orbit will be attracting, that is asymptotically stable, if on the average along the orbit it is locally stable. If neighboring points diverge away from an orbit, the corresponding behavior will be sensitive to small perturbations. In this case, the instability will amplify the perturbations and the orbit will be locally unstable, even if the orbit initially converged toward some attractor.

In the case of $1 \mathrm{D}$ maps, these stability criteria are measured directly by the slope of the map at points visited by an orbit. In particular, if the slope at a point is less than one, nearby points will be brought closer to it at the next iteration of the map. Similarly, if the slope at a point is greater than one, nearby points will be spread apart under iteration of the map. An asymptotically stable orbit, then, requires the geometric average of these slopes along the orbit to be less than one. When this average is greater than one the orbit is unstable and, consequently, initially small deviations from the orbit will increase under iteration of the map.

This sensitivity to small deviations has important consequences for the physical behavior associated with chaotic dynamical systems. For if these deviations are due to some initial uncertainty in specifying or measuring a state, then this uncertainty will grow (exponentially, at first) until one can no longer predict the state of the system within the attractor. The information about the initial state of the system is lost in a finite amount of time and so the system is effectively unpredictable $[18,19]$. This sensitivity to small errors can be considered one of the defining features of chaos [20]. This in turn leads to a physical interpretation of the degree of randomness, given by the average local stability, as the rate at which information about states is lost $[18,19]$.

The measure of average local stability, the Lyapunov characteristic exponent $\lambda$, can be expressed in two different, but related, ways [21]. The first is the information-theoretic entropy, given in the case of $1 \mathrm{D}$ maps [19] by

$$
\lambda(r)=\int_{0}^{1} P(x) \ln \left|f^{\prime}\left(r, x_{n}\right)\right| \mathrm{d} x
$$


where $P(x)$ is the asymptotic probability distribution of an orbit at a given parameter value, such as shown in fig. 2, and $f^{\prime}$ is the slope of the map. If one assumes ergodicity of the orbit within the attractor, there is an alternative form for computing $\lambda(r)$. It is given by

$$
\lambda(r)=\lim _{N \rightarrow \infty} \frac{1}{N} \sum_{n=0}^{N} \ln \left|f^{\prime}\left(r, x_{n}\right)\right| .
$$

With this definition the measure of average local stability is such that if

(i) $\lambda(r)<0$, the orbit is stable and periodic;

(ii) $\lambda(r)=0$, the orbit is neutrally stable;

and (iii) $\lambda(r)>0$, the orbit is locally unstable and chaotic.

Many of the features found in the bifurcation diagram of fig. 1 are reflected in the curve of the characteristic exponent as a function of $r$. This curve is shown in fig. 3 for the parameter regime of fig. 1 . For $r<r_{c}, \lambda(r) \leq 0$, indicating the existence of periodic orbits only. In this regime, the period-doubling "pitchfork" bifurcations occur where $\lambda$ vanishes. That is, an orbit must first pass through a neutrally stable attractor before it can take on a qualitatively different structure. Between these bifurcations, $\lambda$ approaches $-\infty$ as the critical point $x_{c}$ becomes a point on the periodic orbit. The orbit is said to be superstable. The logarithmic divergence of $\lambda$ at this value of the parameter is easily inferred from eq. $(2-10)$ or (2-11) because the slope at the critical point vanishes (see appendix B). The relaxation of initial transients onto the periodic orbit differs on either side of these superstable bifurcations. For $r$ less than the bifurcation value $r_{\text {superstable, the approach of an initial transient is (eventually) from only one side; }}$

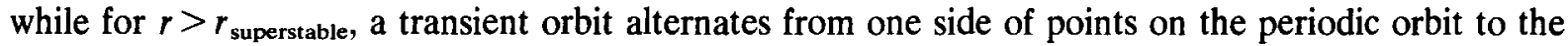
other. For example, the period 1 orbit becomes superstable at $r=2$. For $r<2$, any initial condition will approach from the left of $x_{\mathrm{c}}$, even though it may have started from the right half of the interval. For $r>2$, initial transients alternate sides as they approach the periodic orbit. The period 2 orbit which becomes superstable at $r=3.236068 \ldots$ consists of two points. The transients in this case for $r<$ $r_{\text {superstable }}$ eventually approach only from the lower side of each point, while for $r>r_{\text {superstable }}$ they approach the points of the attractor from each side. The change in stability associated with the superstable bifurcation is clear in the $\lambda(r)$ curve, despite the fact that it is not at all visible in the bifurcation diagram of fig. 1 .

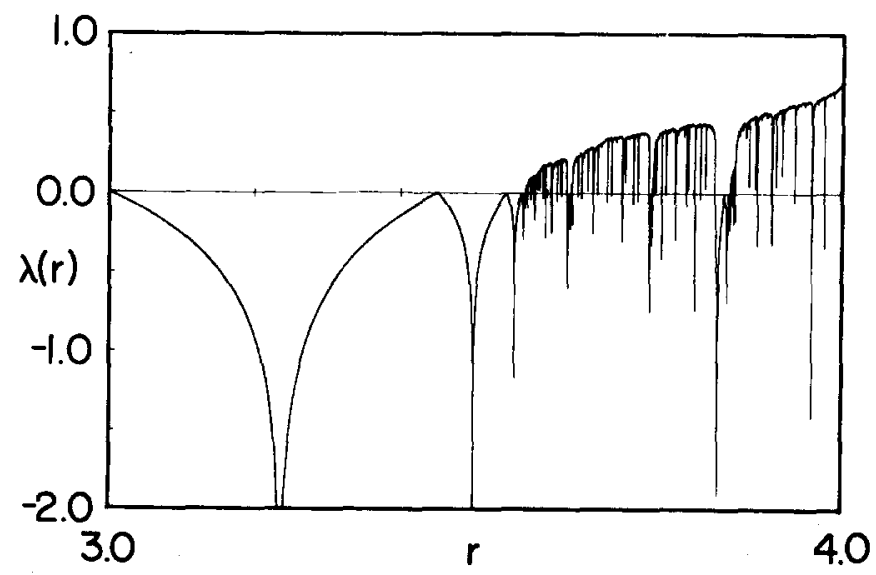

Fig. 3. Characteristic exponent versus bifurcation parameter for the logistic equation calculated using eq. (2-11) for 30000 iterations at each of 7000 increments of $r$ in $[3,4]$. 
Finally at $r=r_{c}, \lambda=0$ and one sees a bifurcation to chaotic behavior indicated by positive $\lambda$ for $r>r_{\mathrm{c}}$. Huberman and Rudnick [22] have recently shown that the envelope of $\lambda$ near $r_{\mathrm{c}}$ displays universal behavior reminiscent of an order parameter near the critical point of a phase transition. That is, one can write

$$
\lambda(r)=\lambda_{q}\left(r-r_{\mathrm{c}}\right)^{\tau}
$$

with $\tau=\ln (2) / \ln (\delta)=0.4498069 \ldots$ and $\lambda_{q}$ a constant. Cascades of higher periodicities in the chaotic regime are indicated by windows of negative $\lambda$ in which $\lambda$ goes through a period-doubling sequence of pitchfork and superstable bifurcations. Above the accumulation point in each window the envelope of positive $\lambda$ scales as in eq. (2-12) except that the constant $\lambda_{q}$ depends on the periodicity $q$ and the width of the window. From numerical studies of the characteristic exponent using eq. (2-11), we have verified this scaling behavior for various periodicities. For example, for $q=1,3$ and $5, \tau=0.45+0.01$, and $\lambda_{1}=0.84, \lambda_{3}=1.7$ and $\lambda_{5}=1.6$. Figure 4 shows $\lambda(r)$ and eq. (2-12) graphed with the values for $q=1$ and 3.

An estimate of $\lambda_{q}$ can be based on the observation that at band joinings $\lambda$ is inversely proportional to the number of bands. Specifically, at a bifurcation from $q \cdot 2^{n}$ to $q \cdot 2^{n-1}$ bands, the characteristic exponent is given by

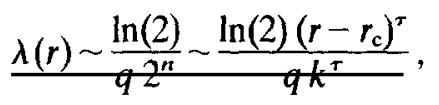

that is,

$$
\lambda_{q} \sim \frac{\ln (2)}{q k^{\tau}},
$$

where the approximation is less than a few percent and $k$ is the constant of proportionality of (2-9) which depends on the periodicity $q[23]$.

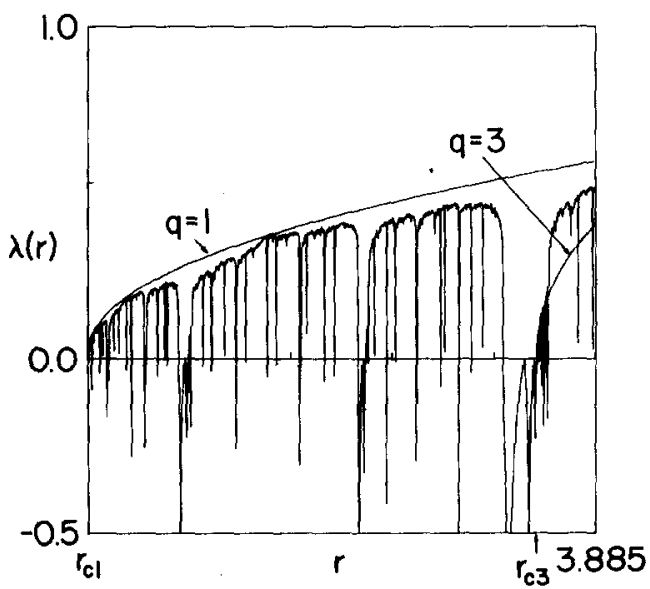

Fig. 4. Characteristic exponent curve $\lambda(r)$ from fig. 3 on an expanded scale from $r_{c}$ to $r=3.885$. The two smooth curves show the fit of eq. (2-12) to the envelope of the $q 2^{n}$ cascades. For $q=1$ and $q=3$, we find $\lambda_{1}=1.01$ and $\lambda_{3}=1.7$ in fitting eq. (2-12). The curve for the $q=3$ chaotic regime has been extended beyond its range of validity to make it easier to see its fit to the envelope of positive characteristic exponent. 
When one looks for the feature in $\lambda(r)$ corresponding to bands merging the self-similarity again becomes apparent in the $\lambda(r)$ curve of fig. 3. There is a peculiar upturn of $\lambda(r)$ toward $\ln (2)$ near $r=4.0$ [24]. This upturn also displays a critical behavior given by [10]

$$
\lambda(r) \sim 1-b(4.0-r)^{1 / 2}
$$

where $b$ is a constant. In this regime, there is a conspicious absence of negative dips in $\lambda(r)$ indicating the existence of little observable periodic behavior. These features also occur for values of $r<4.0$ at which bands merge. Several of the corresponding "steps" in $\lambda(r)$ can be seen in fig. 3 . One of them is shown magnified in fig. 5 near the merging of two bands into one. Using eqs. (2-13) and (2-15), we obtain the following expression for $\lambda(r)$ near band joinings

$$
\lambda(r)=\lambda_{q}\left(r-r_{\mathrm{c}}\right)^{t}\left(1-b_{q}\left(r_{\mathrm{band}}-r\right)^{1 / 2}\right)
$$

where $b_{q}$ depends on the periodicity $q$. Thus, as one would expect, the entire $\lambda(r)$ curve exhibits the same type of self-similarity as the bifurcation diagram. $\lambda(r)$ in each of these self-similar subintervals is very near one-half of that in the preceding subinterval. Again, knowledge of the structure of the $\lambda(r)$ curve in $\left[r_{2-1}, 4.0\right]$ is sufficient to (qualitatively) determine the entire curve for $\left[r_{c}, 4.0\right]$.

Although $\lambda(r)$ is continuous in the chaotic regime, it does not converge to a limit curve with increasing resolution in $r$. It is a curve of infinite length that admits of no closed form representation. Some of the features associated with this property can be described by different types of self-similarity. Furthermore, these self-similar features can be described by a "fractal" dimension [16].

To emphasize the unusual nature of the $\lambda(r)$ curve, we shall mention three occurrences of self-similar structure. The first is the global self-similarity found in the bifurcation diagram. The features between $r_{2-1}$ and $r=4.0$ are repeated on smaller scales (reduced by a factor of $\delta$ ) as one approaches $r_{\mathrm{c}}$ from above. This self-similarity has already been discussed above. Another property of the $\lambda(r)$ curve in the chaotic regime is that at every degree of resolution in $r$, there are windows of periodic behavior corresponding to the negative dips in $\lambda(r)$. The width of the windows rapidly decreases with increasing periodicity $q$. The number of windows observed at a given resolution in $r$ appears, from our numerical

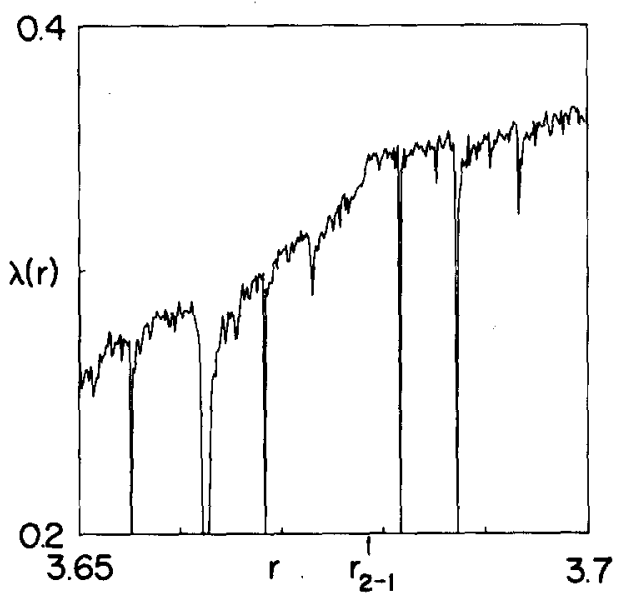

Fig. 5. $\lambda(r)$ near two bands merging into one at $r_{2-1}=3.67857 \ldots$. The kink in $\lambda(r)$ at $r_{2-1}$ as seen in the figure is characteristic of bands merging. Note that $\lambda\left(r_{2-1}\right) \sim \ln (2) / 2 \sim 0.35$. This picture is taken from the data of fig. 3 . 
work, to be roughly independent of the resolution at which $\lambda(r)$ is studied. This is the second self-similar feature of $\lambda(r)$ : a local self-similarity. It depends on the parameter $r$ and gives a measure of the density of periodic windows in the chaotic regime.

These two features, and the self-similar character they lend to $\lambda(r)$, can be summarized by the fractal dimension of the $\lambda(r)$ curve itself. We have measured the fractal dimension of $\lambda(r)$ for $r$ in $\left[r_{c}, 4\right]$ using a standard algorithm discussed by Mandelbrot [16]. The fractal dimension of a curve describes how the curve's length increases when measured on smaller scales. To apply this to the curve of fig. 3, we measure the length of $\lambda(r)$ in units of $s \Delta r$, where $s$ is some multiple of the increment $\Delta r$ in parameter used in making fig. 3. The fractal dimension $\beta$ is obtained then by varying the size of the measuring unit. The number $N$ of measuring units of size $s \Delta r$ is given by

$$
N=A s^{-\beta},
$$

where $A$ is some constant dependent on $\Delta r$. The results of these length measurements are shown on a $\log -\log$ plot in fig. 6. We have taken $s=2^{n}, n=0,1, \ldots, 11$, and found $\beta=1.69$ and $A=4.63 \times 10^{5}$ for $r$ in $\left[r_{\mathrm{c}}, 4\right]$. The fact that $b$ is not 1 , as would be the case for a simple smooth curve, indicates that as the measuring unit is decreased by 2 (say), more than twice as many units are required to cover $\lambda(r)$. Thus, as the resolution is increased more features in $\lambda(r)$ become apparent, so that in the limit the length, $l=N s \Delta r$, of $\lambda(r)$ is infinite.

The last self-similar feature we shall mention is the fractal dimension of the attractors themselves. Periodic orbits and chaotic attractors have trivial fractal dimensions 0 and 1 , respectively. The former consist of a countable number of discrete points and the latter fill out dense bands in the interval. At the transitions to chaos, however, where $\lambda(r)$ vanishes, the attractors have a self-similar structure: the uncountable number of points on these orbits are distributed by factors of Feigenbaum's spatial scaling constant $\alpha$. The fractal dimension of these attractors has recently been calculated to be $d_{\text {transition }}=$ $0.538 \ldots[25]$.

These self-similar features are substantially modified in the presence of fluctuations. The fractal dimension of each, though, still gives a qualitative measure of the level at which fluctuations truncate the self-similar structure. We shall return to this point at the end of the following section.

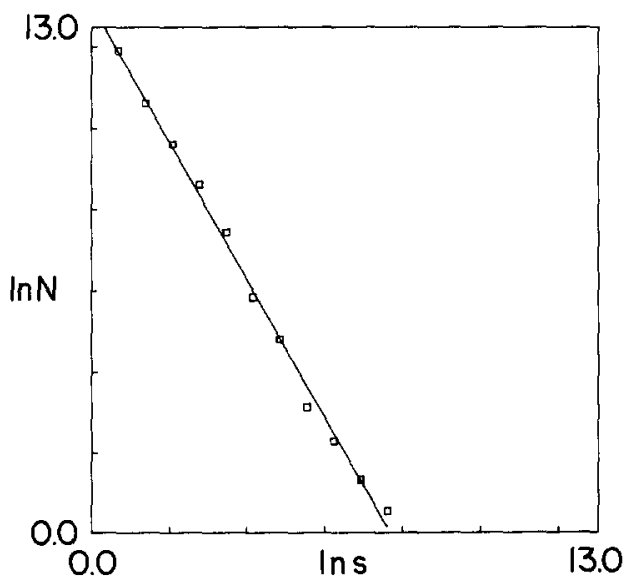

Fig. 6. Log-log plot of the length of the $\lambda(r)$ curve, for $r$ in $\left[r_{c}, 4\right]$ versus the scale of measurement $s . N$ is the number of measuring units of length $s \Delta r$ necessary to cover $\lambda(r)$, where $\Delta r$ is the increment in $r$ used to compute $\lambda(r)$ in fig. 3. According to eq. (2-17), the slope of the line is the fractal dimension $\beta$. For $r$ in the chaotic regime $\left[r_{\mathrm{c}}, 4\right]$ we have found the fractal dimension $\beta=1.69$. 
As a concluding remark, we should point out that self-similarity also leads to scaling predictions relating to the behavior of the power spectra associated with deterministic noise [26]. Once again, one can associate universal exponents with both the growth of deterministic noise as a function of the control parameter and the width of the bands past the bifurcation cascade.

This ends our review of the dynamics of $1 \mathrm{D}$ maps in the absence of fluctuations. In this section, we have emphasized the self-similarity of the dynamics as a function of the bifurcation parameter $r$. In the following sections we shall discuss how this self-similarity allows for a scaling description of the effect of fluctuations on the cascade bifurcation. In particular, we will study the effect of noise on the fractal attractor at $r_{\mathrm{c}}$ and will find that noise can be described by a scaling theory, such as found in the theory of critical phenomena. This noise scaling is also of interest in the study of the effect of noise on the fractal structure of chaotic attractors in higher dimension, even away from period doubling bifurcations.

\section{Dynamics in the presence of fluctuations}

The nature of the fluctuations introduced into a dynamical model depends on the coupling between the physical process to be described and the source of random perturbations. In describing a physical system specified by a set of differential equations, dynamical systems theory considers the action of a flow on a suitable phase space of states. From this perspective fluctuations can enter in two ways: first, as an "external" stochastic force that perturbs the phase space trajectory; and second, as a random perturbation of the parameters specifying the flow itself. We shall call the first additive noise and the second parametric noise. The analog of additive noise for 1D maps is of the following form,

$$
x_{n+1}=f\left(r, x_{n}\right)+p_{n}
$$

where $p_{n}$ represents a random deviation from the deterministic orbit. For parametric noise, the fluctuations perturb the form of the nonlinear function or the parameters in the map. For example, if we write the deterministic equation as

$$
x_{n+1}=r f\left(x_{n}\right)
$$

with $r$ as the bifurcation parameter, then parametric fluctuations would be of the form

$$
x_{n+1}=\left(r+q_{n}\right) f\left(x_{n}\right)
$$

or

$$
x_{n+1}=r f\left(x_{n}\right)+q_{n} f\left(x_{n}\right)
$$

with $q_{n}$ representing the fluctuations in the parameter $r$. In the case of $1 \mathrm{D}$ maps, the two physically distinct types of fluctuation reduce to basically the same form, namely that of eq. (3-1), except that eq. (3-4) has different statistics for the "external" stochastic force: $q_{n} f\left(x_{n}\right)$ rather than just $p_{n}$. This will introduce higher-order correlations, but for small fluctuations the systems of eqs. (3-1) and (3-4) will exhibit the same behavior. In what follows we shall discuss the effect of changes in the level of fluctuations on the behavior of eq. (3-1) with $f(r, x)=r x(1-x)$. We shall take $p_{n}$ as a Gaussian or 


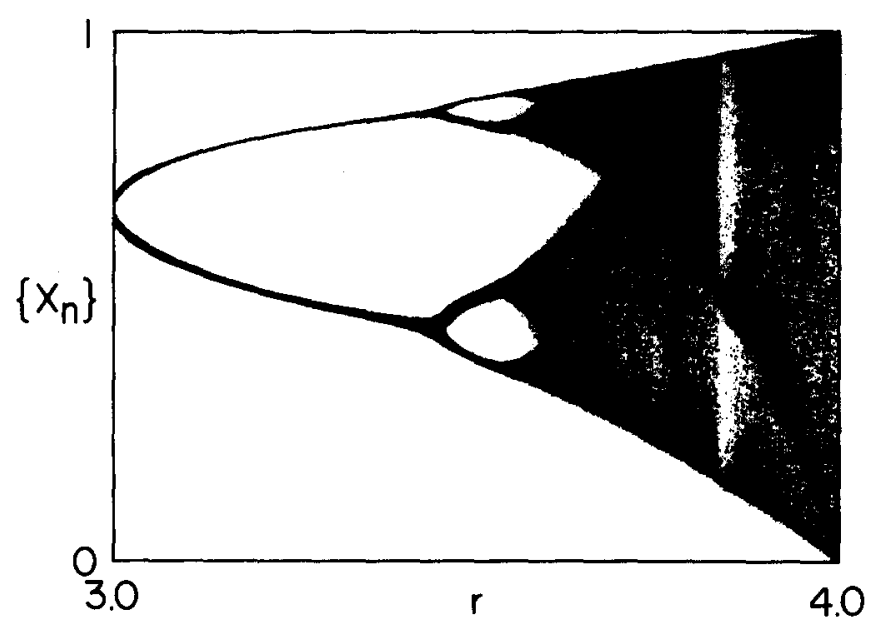

Fig. 7. Bifurcation diagram in the presence of zero-mean Gaussian fluctuations with standard deviation, or noise level, $\sigma=10^{-3}$. Computational details are the same as in fig. 1, except that eq. (3-1) was used.

uniform random variable with standard deviation $\sigma$ and zero mean. We have found that the results do not depend significantly on the choice of distribution. We will refer to $\sigma$ as the noise level of the fluctuations.

A heuristic description of fluctuations, as imposing a minimum scale of deterministic resolution, léads one to properly anticipate the bifurcation diagram in the presence of noise. Figure 7 illustrates the effects of noise on the 1D map, with $p_{n}$ a Gaussian random variable of standard deviation $\sigma=10^{-3}$. As can be seen in comparing figures 1 and 7 , the fluctuations truncate the detailed structure by smearing the sharp features of the probability distribution of the attractors seen in fig. 1. An example of this effect on two bands is shown in fig. 8 for $r=3.7$ and $\sigma=10^{-3}$. This should be compared to fig. 2. The periodic regime, where the orbits are slightly broadened, is easily distinguished from the chaotic regime, where the distribution of points within the bands appears more uniform than in fig. 1. When fluctuations of sufficient amplitude are added, periodic orbits broaden into bands similar to chaotic attractors. As one would expect, the reverse process of chaotic attractors turning into periodic orbits does not happen.

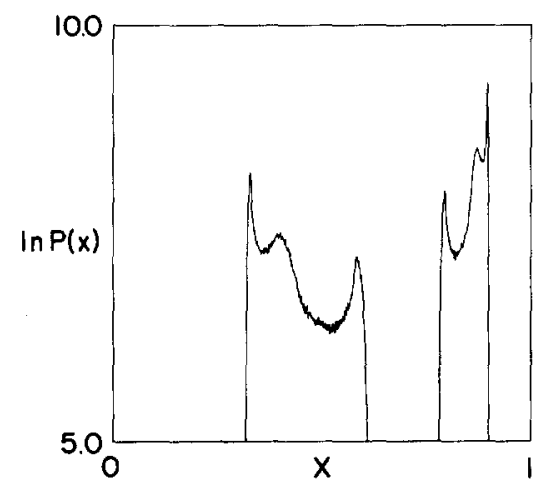

Fig. 8. Logarithmic plot of the (unnormalized) probability density $P(x)$ of the two band attractor at $r=3.59687$ in the presence of $\sigma=10^{-3}$ noise as in fig. 7. Compare this with fig. 2 in which there is no added noise. $P(x)$ is a histogram of $10^{6}$ iterations of eq. (3-1) with $p_{n}$ a uniformly distributed random variable of standard deviation $\sigma=10^{-3}$. The points have been partitioned into $10^{3}$ bins. 
In other words, fluctuations generally increase the degree of randomness or chaos in the dynamics, while destroying the periodic windows encountered in the chaotic regime. This enhanced "observability" of the chaotic behavior in the presence of fluctuations originates in the local instabilities underlying the deterministic chaos. Thus fluctuations affect the local stability properties of the attractors, while leaving their global stability relatively unchanged.

Some of the structure observed in the deterministic limit $(\sigma=0)$ is still visible above $r_{\mathrm{c}}$ when noise is added. In particular, it is evident from fig. 7 that in the chaotic regime a period three orbit is still present, although, windows of higher periodicity are not. The cascades of higher periodicity become unobservable at noise levels whose intensities are proportional to the parameter width of the window. Also, as shown in figures 7 and 8, the images of the critical point still appear as regions of higher probability, but with fewer of the higher iterates encountered in the deterministic limit. Furthermore, for the noise level of fig. 7, a period of four is the highest that remains in the primary $2^{n}$ cascade, while all higher periods are washed out.

To summarize the effects of noise for a given cascade, we will define $\sigma_{p}$ as the noise level that results in a maximum observable period $p$. This quantity determines a lower bound on those periods which have been washed out. The effect on the primary $2^{n}$ cascade is illustrated by a diagram, shown in fig. 9 , displaying a gap in the observable periods which increases with noise level. This gap represents the set of attractors (both periodic orbits and chaotic bands) of periods greater than $p$ which are inaccessible at a particular noise level $\sigma_{p}$. Figure 9 shows the qualitative dependence of the gap on the noise level, where the vertical axis denotes the period $p=2^{n}$ of periodic orbits or the number of bands $p=2^{n}$ of the chaotic attractor. The deterministic limit $\sigma=0$ corresponds to the full cascade bifurcation. With increasing noise level, though, a symmetric gap in the bifurcation sequence appears, rendering unobservable successively more periodic and chaotic bifurcations. The gap in fig. 9 symbolizes a set of forbidden attractors whose periods cannot be observed at a given noise level. Both the largest

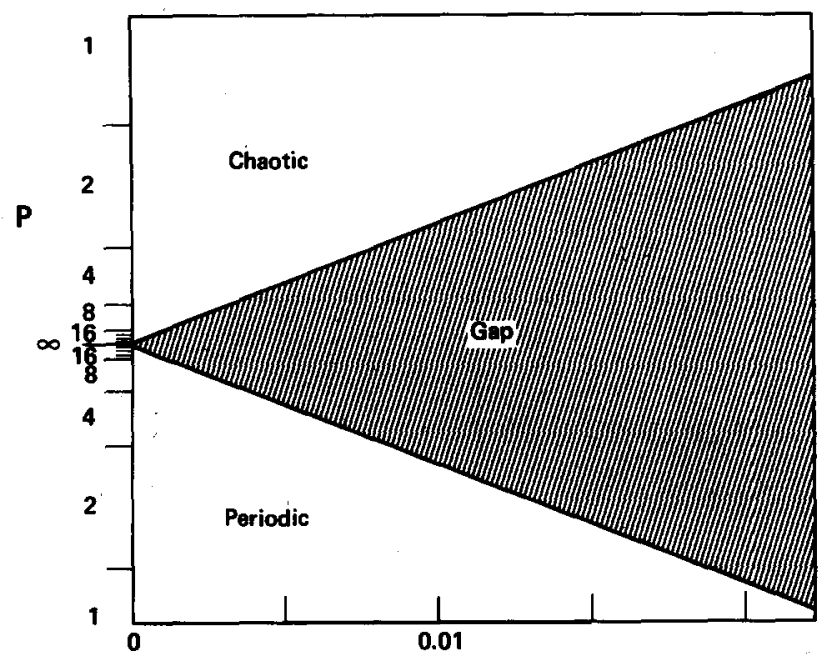

Fig. 9. The set of observable attractors for the cascade bifurcation as a function of (normalized) noise level. The vertical axis denotes the period $p$ of an attractor given (i), in the case of a driven oscillator [1], by the ratio of the response period to the period of the driving force, or (ii), in general, by the period (number of bands) of the periodic (chaotic) attractor. The noise level $\sigma$ along the horizontal axis corresponds to the standard deviation of thermal-like fluctuations in which the system is immersed. The shaded area represents the set, or "gap", of unobservable attractors obliterated by a given level of fluctuations. 
observable period and maximum number of observable bands decrease with increasing noise level $\sigma$. As an example of the alteration of the cascade bifurcation with noise level, consider starting in fig. 9 from a period 4 orbit at $\sigma=0$. As the noise level is increased from this point, the gap is finally reached at $\sigma \sim 5 \times 10^{-3}$ where the attractor appears almost like a 4 band chaotic attractor. The attractors observed at higher noise levels are those along the upper boundary of the gap, within the chaotic regime. That is, increasing noise levels strengthen the band-like character of the attractor, eventually inducing a transition to a chaotic attractor. This transition from a noisy periodic attractor to a chaotic one corresponds to a vertical jump across the gap. The attractor reaches another transition point at $\sigma \sim 10^{-2}$ where the fluctuations have become strong enough to broaden the four chaotic bands into two bands. A similar transition takes place, in principle, at $\sigma \sim 2 \times 10^{-2}$ where the 2 band attractor will broaden into one band. Similarly, at a fixed noise level in fig. 9, one encounters a transition to a chaotic attractor at parameter values which decrease with increasing noise level. Thus the existence of the gap also illustrates the fact that fluctuations can induce a transition to chaos at a lower threshold.

Since the exact values of $\sigma$ and $p$ for which a gap appears depends on the determination of when a given period becomes unobservable with increasing noise, we have calculated the Lyapunov characteristic exponent curve to provide a consistent measure of the onset of chaos as a function of noise level. When noise is added, the distinction between periodic orbits and chaotic attractors is no longer as straightforward as in the deterministic limit: all attractors eventually fill out intervals whose width depends on the noise level. Nevertheless, there is a qualitative difference between the stability properties of the two types of noisy attractors, a difference which is reflected in the behavior of the characteristic exponent. In calculating the characteristic exponent in the presence of noise, we used eq. (2-11) with $f^{\prime}$ the derivative of the deterministic map evaluated at points along an orbit $\left\{x_{n}\right\}$ taken from iterates of eq. (3-1).

Figure 10 shows our computation of $\lambda(r)$ at the same noise level as the bifurcation diagram of fig. 7 . When this is compared to the deterministic case, fig. 3, several changes are immediately apparent. First, the small parameter windows of periodic behavior in the chaotic regime disappear. Second, the higher-order period-doubling bifurcations merge into a single smooth curve near $r_{\mathrm{c}}$. Third, bifurcations between periodic orbits, where $\lambda=0$ in the absence of noise, become more stable when fluctuations are added. Fourth, the first transition to positive $\lambda$ occurs earlier in the bifurcation sequence. We will continue to associate this latter feature with the onset of chaotic behavior. And finally, the value of $\lambda$ at parameter values that are chaotic in the deterministic limit is effected substantially less than for the case

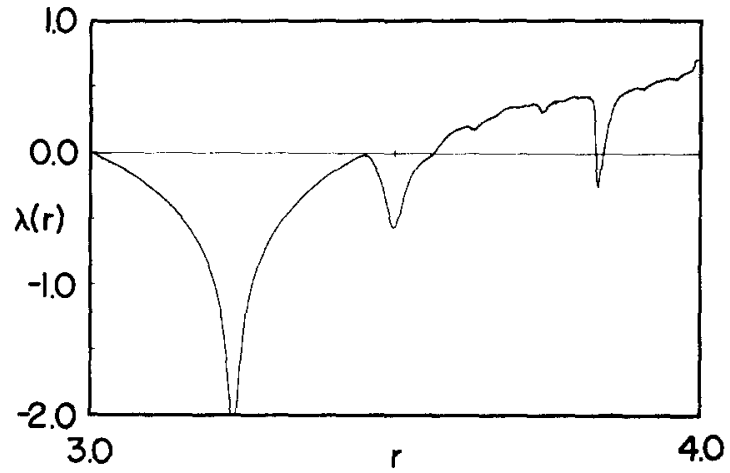

Fig. 10. Characteristic exponent versus bifurcation parameter $r$ at the noise level of fig. $7 ; \sigma=10^{-3}$. Details of the calculation are the same as in fig. 3 , except that eq. (2-11) was used with iterates of eq. (3-1). 
of periodic behavior. In most chaotic regimes the addition of noise leaves $\lambda$ unchanged over some range of noise level.

Figures 7 and 10 were constructed using additive noise at fixed parameter values. A study of these figures makes it clear that it is difficult to anticipate the effect of adding noise unless the behavior of the deterministic system is known at adjacent parameter values. Roughly speaking, adding fluctuations alters a deterministic orbit so that it wanders over points on the attractors at adjacent parameter values. This suggests a simple model for the action of noise on the deterministic behavior: the effect of fluctuations is to average the structure of deterministic attractors over some range of nearby parameters. That is to say, there is an equivalence between a perturbation of an orbit and a perturbation of the map itself, in the sense that at each iteration the effect of a perturbation of an orbit can also be obtained by a suitable change in parameter. With this equivalence, all of the features of the bifurcation diagram, fig. 7, and the $\lambda(r)$ curve, fig. 10, can be understood, as well as other consequences of adding noise.

To understand why this averaging of parameters is effective, consider an orbit $\left\{x_{n}\right\}$ of eq. (3-1), generated by some particular sequence of fluctuations $\left\{p_{n}\right\}$. If just the right sequence of fluctuations $\left\{q_{n}\right\}$ occurs, an identical trajectory will be produced by a parametric noise process of the form of eq. (3-3) or (3-4). This sequence $\left\{q_{n}\right\}$ can be found by equating the trajectories step by step, that is,

$$
x_{n+1}=r f\left(x_{n}\right)+p_{n}=\left(r+q_{n}\right) f\left(x_{n}\right) .
$$

For the case $f(x)=x(1-x)$ we obtain the following equation for $q_{n}$

$$
q_{n}=\frac{p_{n}}{x_{n}\left(1-x_{n}\right)}
$$

That is, at each iteration the effective parameter value is $r^{\prime}=r+q_{n}$. For this particular iteration, the noisy system behaves just like a purely deterministic system at parameter value $r^{\prime}$, except that the point $x_{n}$ is not necessarily on the attractor for this parameter. Therefore, the attractor of the noisy system can be approximated by an appropriately weighted average of the deterministic attractors at nearby parameter values.

As may be seen from eq. (3-6), the statistical properties of $\left\{q_{n}\right\}$ are quite different from those of $\left\{p_{n}\right\}$. Since $q_{n}$ depends on $x_{n}$, its statistics may be as complicated as those of $x_{n}$. For example, suppose the deterministic attractor is a period 2 orbit and the additive fluctuations $p_{n}$ are small. Because the two points of the orbit have different values, eq. (3-6) says that the fluctuations $q_{n}$ with odd $n$ will on the average have a different magnitude than those with $n$ even. Thus, in this case, if the additive fluctuations are ergodic, the parametric fluctuations are not. In addition, $\left\{q_{n}\right\}$ need not be Gaussian, even if $\left\{p_{n}\right\}$ is.

To calculate the statistical properties of $\left\{q_{n}\right\}$ exactly would require simulating the orbit $\left\{x_{n}\right\}$. Nevertheless, very crude estimates of a few moments of $q_{n}$, together with a knowledge of the deterministic bifurcations, provide a good understanding of the effect of additive noise. In what follows, we will estimate the first two moments of the equivalent parametric noise $q_{n}$. We will then discuss how this can be used to explain the observed effects of noise on figures 1 and 3 .

We will now compute the average of $q_{n}$. Since the fluctuations $p_{n}$ are statistically independent of $x_{n}$ we can write 


$$
\left\langle q_{n}\right\rangle=\left\langle p_{n} /\left(x_{n}\left(1-x_{n}\right)\right)\right\rangle=\left\langle p_{n}\right\rangle\left\langle 1 /\left(x_{n}\left(1-x_{n}\right)\right)\right\rangle=0
$$

where $\langle\ldots\rangle$ denotes time average and where we have assumed $\left\langle p_{n}\right\rangle=0$. Thus the average of $q_{n}$ is zero.

Estimating the second moment is more difficult. Squaring eq. (3-6) and again making use of the fact that $p_{n}$ and $x_{n}$ are uncorrelated, we obtain

$$
\left\langle q_{n}^{2}\right\rangle=\left\langle p_{n}^{2}\right\rangle\left\langle 1 /\left(x_{n}\left(1-x_{n}\right)\right)^{2}\right\rangle,
$$

which, unfortunately, depends on an unknown moment of $x_{n}$. However, in many cases this moment can be estimated. For example, if the deterministic orbit approaches a fixed point, and the additive noise is small, the value of the fixed point can be used to obtain a good estimate of $\left\langle q_{n}^{2}\right\rangle$. To do this for a limit cycle, the fluctuations about each point on the deterministic orbit must be considered separately, since in this case the fluctuations $q_{n}$ are not ergodic.

A simpler, but less accurate, expression may be obtained by rewriting $x_{n}\left(1-x_{n}\right)$ in terms of $x_{n+1}$,

$$
x_{n}\left(1-x_{n}\right)=\left(x_{n+1}-p_{n}\right) / r .
$$

Squaring, substituting this into eq. (3-8), and expanding the denominator, we obtain

$$
\left\langle q_{n}^{2}\right\rangle=\left\langle\left(r p_{n} / x_{n+1}\right)^{2}\right\rangle\left\langle 1-2 p_{n} / x_{n+1}+3\left(p_{n} / x_{n+1}\right)^{2}-\cdots\right\rangle
$$

with $p_{n}$ and $x_{n+1}$ correlated. If $p_{n}$ is small, however, the dominant factor determining $x_{n+1}$ is $x_{n}$, and $p_{n}$ will be approximately uncorrelated with $x_{n+1}$. With this assumption, we find

$$
\sigma_{q} \sim r \sigma_{p}\left\langle 1 / x^{2}\right\rangle^{1 / 2}
$$

where $\sigma_{q}$ and $\sigma_{p}$ are the standard deviations of $q_{n}$ and $p_{n}$, respectively. To estimate $\left\langle 1 / x^{2}\right\rangle$, we define $\Delta x^{2}=x^{2}-\left\langle x^{2}\right\rangle$. If $\left\langle\Delta x^{2}\right\rangle \ll\left\langle x^{2}\right\rangle$, then $\left\langle 1 / x^{2}\right\rangle$ can be expanded to give

$$
\sigma_{q} \sim \frac{r \sigma_{p}}{\left(x^{2}\right)^{1 / 2}}\left(1-\left\langle\frac{\Delta x^{2}}{\left\langle x^{2}\right\rangle}\right\rangle\right)^{1 / 2}
$$

which is a good approximation for periodic orbits. For chaotic attractors there is no reason to assume $\left\langle\Delta x^{2}\right\rangle \ll\left\langle x^{2}\right\rangle$ unless the probability density is sharply peaked or consists of bands. From numerical calculations, however, the moments in eqs. (3-8), (3-11) and (3-12a) all agree to within less than a percent for $r$ in $[3,4]$.

In practice, then, very coarse approximations are effective. For example, if we take $r=4.0$ where the chaotic attractor fills the entire interval, approximate $\left\langle x^{2}\right\rangle^{1 / 2}$ by its median value of 0.5 , and take $\Delta x^{2}=0$, we can obtain a crude approximation from eq. (3-12a) for the standard deviation of the equivalent parametric noise level $\sigma_{q}$ for a given additive noise level $\sigma_{p}$. This estimate is given by

$$
\sigma_{q}=8 \sigma_{p}
$$

With this equivalence, it is possible to construct a good approximation to the noisy bifurcation diagram of fig. 7 from the deterministic bifurcation diagram of fig. 1 . We have the following rule: 
the behavior in the presence of noise can be determined by a weighted average over nearby deterministic dynamics with a distribution in $r$ of standard deviation $\sigma_{q}$. For example, uniform averaging over a fixed range in $r$ yields a good approximation to observed effects of fluctuations. To obtain the bifurcation diagram for fig. 7 from that of fig. 1, the averaging should use a window in $r$ several standard deviations wide. Specifically, the standard deviation for parameter averaging in this case should be $\sigma_{q}=8 \times 10^{-3}$. Appendix $\mathrm{C}$ discusses the use of this rule in more detail.

This rule provides a simple explanation of the bifurcation gap schematically shown in fig. 9. In the deterministic limit, for parameters close to $r_{\mathrm{c}}$, it is possible to resolve a periodic orbit or a chaotic attractor of arbitrarily high period. If noise is added, however, the higher periods become unresolvable. According to the rule, this is due to the noise averaging over adjacent higher and lower period attractors. In particular, the transition to chaos lowers with increasing noise level as the behavior is averaged over a wider range of parameters so that periodic orbits at successively lower parameters are averaged with chaotic attractors.

Above $r_{\mathrm{c}}$, the averaging over nearby parameters smears out all windows of periodic behavior, except the large period 3 window. As has been observed by Mayer-Kress and Haken [27], the effect of noise on the asymptotic probability density of this particular orbit is quite different than the effect of noise on (say) the period 4 orbit in the main period-doubling sequence. The probability density for the period 4 orbit consists of four delta functions in the deterministic limit. As fluctuations are added to the system, these delta functions broaden, with essentially zero density in between them. As more noise is added, this process continues until eventually the peaks merge pairwise so that the period of four is lost, leaving only two bands. The behavior of the period 3 orbit is quite different, as shown in the noise bifurcation diagram of fig. 11. As noise is added, in addition to some thickening of the three delta functions, a broad background suddenly fills in between the peaks. The sequence of probability distributions of fig. 12 illustrate the noise-induced bifurcations in more detail. With further increases in noise level, this background rises until the peaks are eventually washed out, as seen in fig. 12c. The changes in structure of $P(x)$ are also reflected in the characteristic exponent versus noise level curve of fig. 13. The rapid rise of $\lambda$ for small noise corresponds to the broadening of the delta functions of the

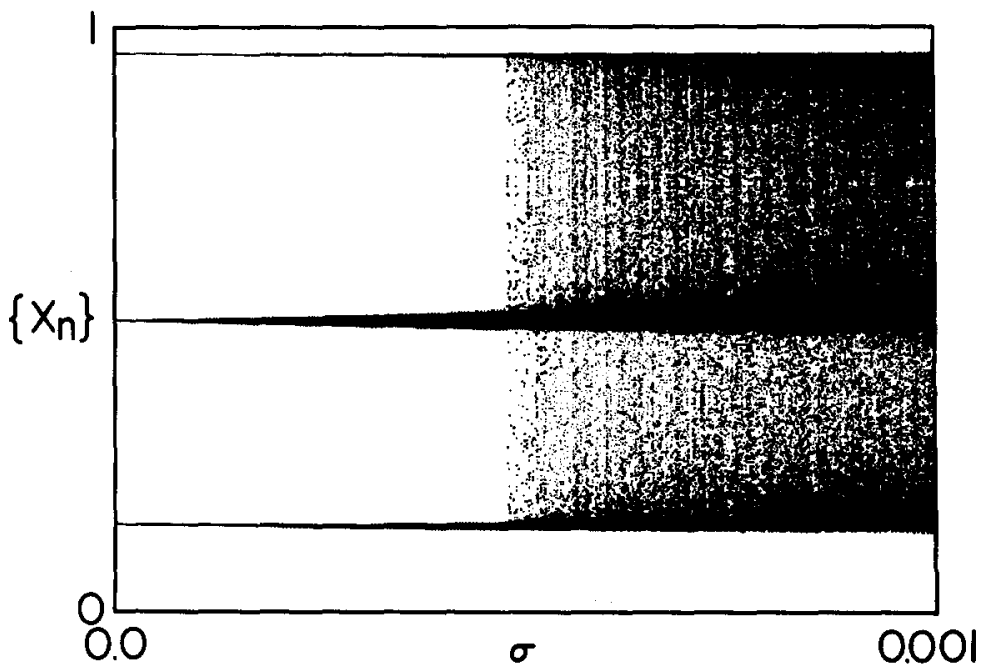

Fig. 11. Noise bifurcation diagram at superstable period 3 orbit: attractor versus noise level $\sigma$ at fixed parameter $r_{3}=3.831874 \ldots 500$ iterates of eq. (3-1) are plotted after an initial 500 iterations at each of the 750 increments in $\sigma$. 

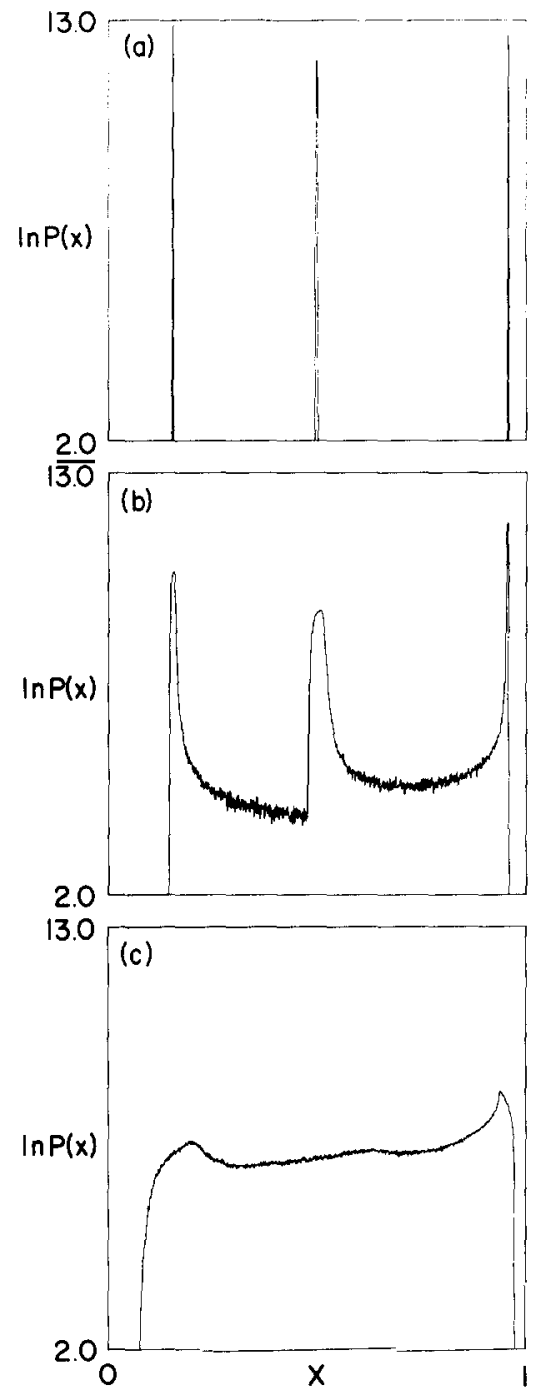

Fig. 12. Log plot of (unnormalized) probability density at the superstable period 3 orbit, $r_{3}=3.831874 \ldots$, for three different noise levels: (a) $\sigma=10^{-4}$, (b) $\sigma=10^{-3}$ and (c) $\sigma=10^{-2} \cdot 10^{6}$ iterates of eq. (3-1) were partitioned into $10^{3}$ bins. (b) shows the probability density as seen in the bifurcation diagram of fig. 7. The noise induces a bifurcation from the period three orbit to a single band at a lower noise level than (say) in the $2^{n}$ cascade, as it is closer, in the sense of equivalent parametric noise, to the single large chaotic band at slightly lower parameter.

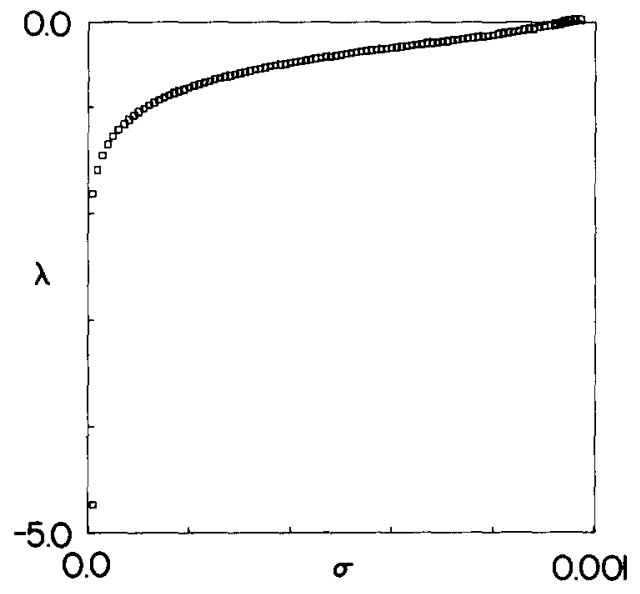

Fig. 13. Characteristic exponent $\lambda$ at the superstable period 3 orbit as a function of noise level $\sigma$. At each noise level, $\lambda$ was calculated with $10^{6}$ iterations of eq. (3-1) using eq. (2-11). The rapid rise in $\lambda$ for very small $\sigma$ corresponds to the broadening of the delta functions of the periodic orbit, while the nearly linear increase in $\lambda$ for moderate $\sigma$ corresponds to the rise of the single band "floor" in $P(x)$ seen in the previous sequence of figures.

deterministic period 3 orbit, while for larger $\sigma$ the nearly linear increase in $\lambda$ reflects the rise of the background "floor" in $P(x)$ seen in figures 11 and 12 .

To understand this effect in terms of equivalent parametric noise, notice that the period 3 orbit is close to a chaotic attractor that is a single band as wide as the period 3 orbit itself. At a sufficiently large noise level this chaotic attractor is averaged with the period 3 orbit, thereby creating the broad background in the probability density. 
In contrast to the sensitivity of periodic behavior to added noise, we find that within various parameter regimes chaotic behavior is relatively insensitive to noise over a wide range of noise levels. As an example of this, consider the single band attractor at $r=3.7$. Figure 14 contains probability distributions showing the attractor at $r=3.7$ in the presence of noise at two different levels. Although increased noise obliterates the detailed delta-function structure in the distributions, the gross features, such as the width and average height of $P(x)$, are unchanged. As a quantitative measure of this insensitivity, fig. 15 shows the characteristic exponent as a function of noise at $r=3.7$. Over a range in noise level similar to the previous figure, there is essentially no change in the characteristic exponent. This behavior is found for many chaotic regimes, although the corresponding range of noise levels depends on the particular band structure. As long as the noise affects only the delta-function structure, the characteristic exponent will change little, but once the noise starts to widen the chaotic bands the exponent will change noticeably.
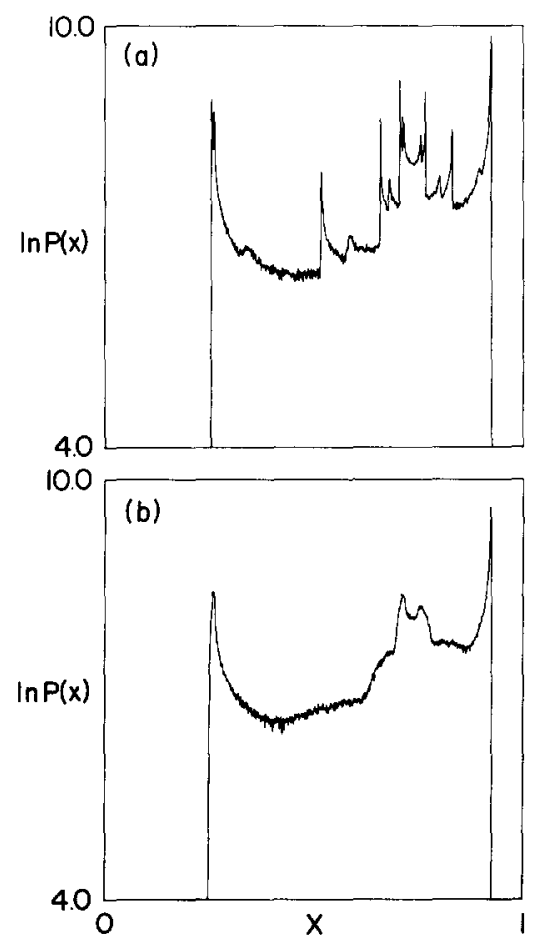

Fig. 14. Logarithmic plot of (unnormalized) probability density for the single band at $r=3.7$ and two noise levels: (a) $\sigma=10^{-4}$ and (b) $\sigma=10^{-3}$. The noise obliterates the detailed structure of the delta functions, but otherwise does not change the width or height of $P(x)$.

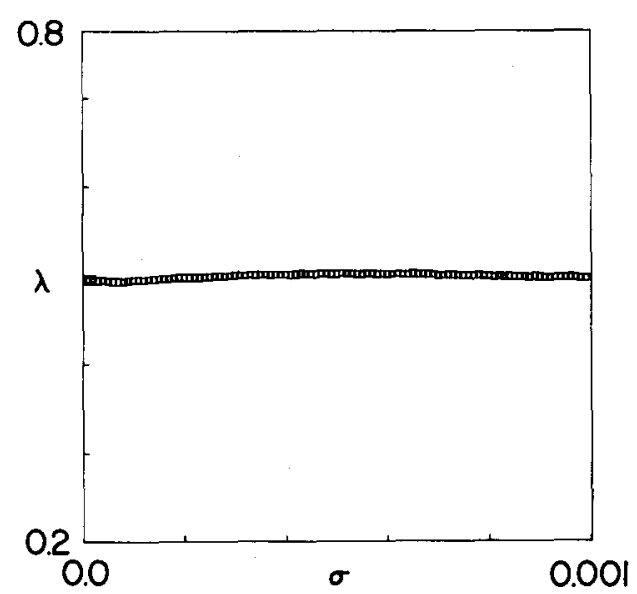

Fig. 15. Characteristic exponent $\lambda$ for the single band at $r=3.7$ as a function of noise level $\sigma$. At each noise level $\lambda$ was calculated with $10^{6}$ iterations of eq. (3-1) using eq. (2-11). As the figure shows, noise affects $\lambda$ very little at chaotic parameter values over wide ranges of noise level. This depends on the degree to which noise changes the probability density (say) by widening it or by filling gaps. For the case of a single band, noise obliterates the peak structure, but this does not contribute significantly to the value of $\lambda$, and so $\lambda$ is relatively independent of noise level.

We can extend the equivalent parametric noise rule to describe the noise-induced changes in $\lambda(r)$. Rather than averaging attractors at adjacent parameter values, we simply average the value of the characteristic exponent at nearby parameters. The deterministic $\lambda(r)$ curve of fig. 3 may be smoothed with a Gaussian distribution of standard deviation $\sigma_{q} \sim 8 \times 10^{-3}$ to obtain an approximation to the $\lambda(r)$ curve of fig. 10. The result of this is shown in fig. 16.

The fractal dimension of the $\lambda(r)$ curve, mentioned at the end of the previous section, provided a 


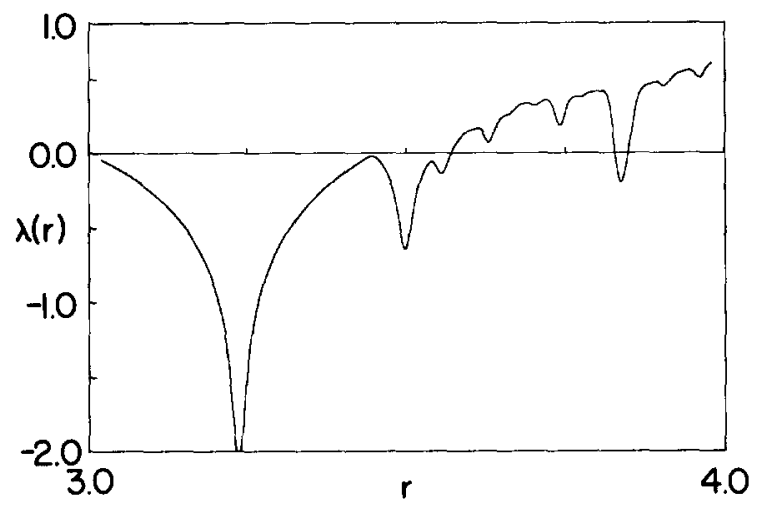

Fig. 16. Gaussian averaging of deterministic $\lambda(r)$ in fig. 3 over data points within two standard deviations. The equivalent parametric noise standard deviation $\sigma_{q}$ was computed from eq. (3-12b); i.e., $\sigma_{q}=8 \sigma_{p}=0.008$. The averaging of $\lambda(r)$ with equivalent parametric noise gives good qualitative agreement.

rough measure of the sensitivity of the self-similar dynamics to the addition of noise. In light of our discussion of the approximate equivalence between additive and parametric noise, we expect that at a given additive noise level $\sigma_{p}$ periodic windows in the chaotic regime of size $\sigma_{q}$ or smaller will be unobservable. Thus, in the sense that the fractal dimension of $\lambda(r)$ quantifies the repetition of bifurcation structure on successively smaller scales, it is also a measure of how this detail is washed out at a given noise level. As a first approximation, the noise level $\sigma$ at which we can no longer observe a window of parameter width $w$ is simply proportional to $w$. If we associate this noise width with the scale of resolution $\Delta r \cdot s$ used to derive eq. (2-17), then the length $l=N \Delta r \cdot s$ of the $\lambda(r)$ curve at a given noise level $\sigma$ is

$$
l \sim \sigma^{-(\beta-1)}=\sigma^{-0.69}
$$

We see, then, that the fractal dimension does yield a qualitative measure of the effect of noise on the detailed structure found in $\lambda(r)$ in the chaotic regime. Before concluding this section, we should also mention a different type of chaotic behavior which is encountered whenever a tangent bifurcation occurs. This new phenomenon, which was originally studied in the context of the Lorenz model by Yorke and Yorke [28] and Pomeau and Manneville [29], is characterized by intermittency, or the appearance of noisy bursts in between laminar or periodic sequences. In what follows, we will consider it in the context of tangent bifurcations in systems exhibiting period doubling behavior [31-33].

Consider the third iterate of the map as given by

$$
F^{(3)}(x)=F(F(F(x)))
$$

with $F=R x(x-1)$ and $R=R_{\mathrm{c}}=1+\sqrt{8}$. For this value of $R$ the map is just tangent to the line $x$ at $x_{\mathrm{c}}=(0.160,0.514,0.956)$. For $R>R_{\mathrm{c}}, F^{(3)}(x)$ passes through the line $x$ giving rise to six new fixed points of which three are stable. This phenomenon of tangent bifurcation is responsible for the way in which the periodic windows appear in the chaotic regime [14]. Now consider eq. (3-14) for $R$ slightly smaller than $R_{\mathrm{c}}$. A sequence of third iterates then would generate both a laminar phase (the iterates move slowly in a staircase fashion close to any of the $x_{\mathrm{c}}$ values) and a chaotic burst as the iterates move erratically under the map before being reinjected into any of the $x_{\mathrm{c}}$ values. 
A number of predictions can be made on the basis of this picture. Expanding $F^{(3)}(x, R)$ about $x_{\mathrm{c}}$ and $R_{\mathrm{c}}$ one has

$$
F^{(3)}(x, R) \cong x_{\mathrm{c}}+\left(x-x_{\mathrm{c}}\right)+a_{\mathrm{c}}\left(x-x_{\mathrm{c}}\right)^{2}+b_{\mathrm{c}}\left(R_{\mathrm{c}}-R\right) \text {. }
$$

Setting $y_{n}=\left(x_{n}-x_{c}\right) / b_{c}$ the recursion relation for the three-fold iterate takes the form

$$
y_{n+1}=y_{n}+a y_{n}^{2}+\epsilon
$$

with $\epsilon=R_{\mathrm{c}}-R$. For the logistic map $a=68.5$. Since the basic step size in the passage near $x_{\mathrm{c}}$ is small the recursion relation near $x_{c}$ is well described by the differential equation

$$
\mathrm{d} y / \mathrm{d} l=a y^{2}+\epsilon .
$$

Integrating this equation for $\epsilon>0$ gives for the number of steps between $y_{\text {in }}$ and $y_{\text {out }}$,

$$
l\left(y_{\text {out }}, y_{\text {in }}\right)=\frac{1}{\sqrt{a \epsilon}}\left[\tan ^{-1}\left(y_{\text {out }} / \sqrt{\epsilon / a}\right)-\tan ^{-1}\left(y_{\mathrm{in}} / \sqrt{\epsilon / a}\right)\right] .
$$

To find the average length of a laminar region, $y_{\text {in }}$ is averaged over the probability $P_{\text {in }}\left(y_{\text {in }}\right)$ of entering some acceptance region $(-G, G)$ and $y_{\text {out }}$ is set equal to $G$. So long as $G \gg \sqrt{\epsilon / a}$, it is given by

$$
\langle l\rangle=\frac{1}{\sqrt{a \epsilon}} \tan ^{-1}(G / \sqrt{\epsilon / a}),
$$

a result obtained if the probability distribution $P_{\text {in }}\left(y_{\text {in }}\right)$ is taken to be uniform over $(-G, G)$.

In order to look for this intermittent route to chaos in the presence of external noise, one has to study

$$
x_{n+1}=R x_{n}\left(1-x_{n}\right)+\bar{\sigma} \xi_{n} .
$$

Here $\xi_{n}$ is a Gaussian random variable with $\left\langle\xi_{n} \xi_{n^{\prime}}\right\rangle=\delta_{n n^{\prime}}$. Proceeding as before, the 3-fold iterate can be represented near the contact point by the Lagevin equation

$$
\mathrm{d} y / \mathrm{d} l=a y^{2}+\epsilon+\sigma \xi .
$$

Here $\xi(l)$ is a Gaussian white noise source such that

$$
\left\langle\xi(l) \xi\left(l^{\prime}\right)\right\rangle=\delta\left(l-l^{\prime}\right)
$$

and $\sigma$ is proportional to $\tilde{\sigma}$. Introducing the corresponding Fokker-Planck equation, one can solve for the average path length in the presence of noise [31]. Here we consider the scaling limit valid for small $\epsilon$. In this limit it can be shown that the average path length in the presence of noise satisfies the scaling relation

$$
\langle l\rangle=\frac{1}{\sqrt{\epsilon}} f\left(\sigma^{2} / \epsilon^{3 / 2}\right) .
$$


As $\sigma^{2} / \epsilon^{3 / 2}$ vanishes, $f$ goes to a constant and we recover the previous result, eq. (3-19). For $\sigma^{2} / \epsilon^{3 / 2} \gg 1$, $f(x) \sim x^{-1 / 3}$ so that in the limit of large noise

$$
\langle l\rangle \sim 1 / \sigma^{2 / 3}
$$

and a chaotic burst as the iterates were erratically under the map before being reinjected into any of the $x_{\mathrm{c}}$ values.

In this section we have described several important effects that additive noise has on the dynamics of the quadratic 1D map. We have also indicated that these can be understood in terms of averaging the dynamics over nearby parameters with an equivalent parametric noise and that the fractal dimension of $\lambda(r)$ gives a qualitative measure of the sensitivity of the bifurcation features to noise. In the next section we shall consider a more quantitative description of the scaling behavior of noise near the accumulation points of period-doubling bifurcations.

\section{Noise as a disordering field}

Several features of the $\lambda(r)$ curve shown in fig. 10 allow the definition of a criterion for the lowering of the chaotic threshold. First, as any amount of noise obliterates at some scale the detailed self-similarity near $r_{\mathrm{c}}$, the first positive transition of $\lambda$ near $r_{\mathrm{c}}$ becomes easier to detect. The transition to chaos (fig. 10) is not only lower than in the deterministic limit (fig. 3), but the slope of the envelope of $\lambda(r)$ at the zero-crossing is no longer infinite. Second, the points of neutral stability $(\lambda \sim 0)$ corresponding to the period-doubling bifurcations below $r_{\mathrm{c}}$, are made more stable by the addition of fluctuations. In fig. 10, for example, the $\lambda(r)$ curve is smoothed and made more negative, indicating greater stability at the points of bifurcation. These two features taken together make the first positive transition of $\lambda$ unambiguous. Hence, we can define the onset of chaos, $r^{+}(\sigma)$, as the value of the parameter $r$ at noise level $\sigma$ for which $\lambda(r)$ first becomes positive. For convenience we will use the normalized parameter $\vec{r}=\left(r-r_{\mathrm{c}}\right) / r_{\mathrm{c}}$. From an extensive study of $\lambda(r)$ versus noise level $\sigma$, in conjunction with the corresponding bifurcation diagrams, we can summarize the effect of fluctuations on the cascade bifurcation in fig. 17, which avoids certain ambiguities associated with fig. 9. Thus we can now follow differing bifurcation paths to the same point in the diagram of fig. 17 unambiguously and without the need for a special transition rule as is the case for fig. 9.

One feature that becomes apparent in the diagram of fig. 17 is the scaling behavior of the onset of chaos $r^{+}(\sigma)$ as a function of noise level $\sigma$. If we denote by $r^{+}(\sigma)$ the value of $r$ for which the characteristic exponent becomes positive at fixed noise level, we can write

$$
r^{+}(\sigma)=k_{q} \sigma^{\gamma}
$$

where $\gamma$ is a critical exponent summarizing the power law behavior of the onset of chaos as a function of noise level and $k_{q}$ is a constant of proportionality depending on the periodicity of the cascade. Using eq. (2-11), we have determined the exponent $\gamma$ and constant $k_{q}$ from numerical experiments to be

$$
\gamma=0.82 \pm 0.01
$$




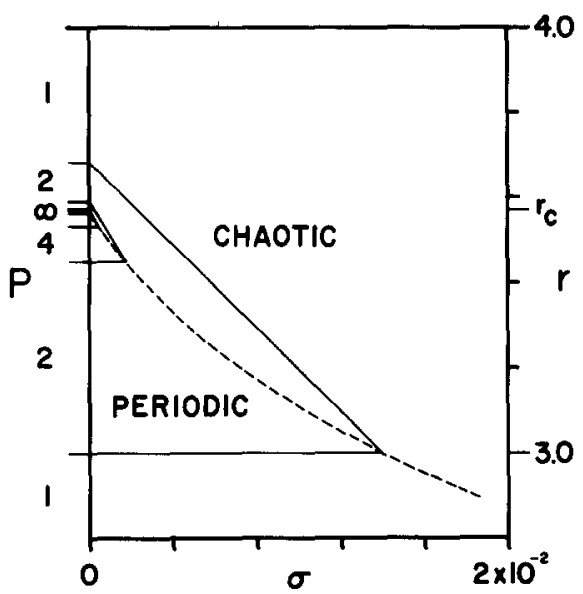

Fig. 17. A rendition of the bifurcation "gap" which does not have any of the associated ambiguities of fig. 9. Period $p$ and bifurcation values of the parameter versus logarithm of the noise level $\sigma$. The dashed line represents $r^{+}(\sigma)$ (defined in text) versus $\sigma$ and indicates that higher noise levels induce an earlier transition to chaos. For numerical details of $r^{+}(\sigma)$ refer to the following figure. Above the dashed line the attractors are chaotic, below periodic.

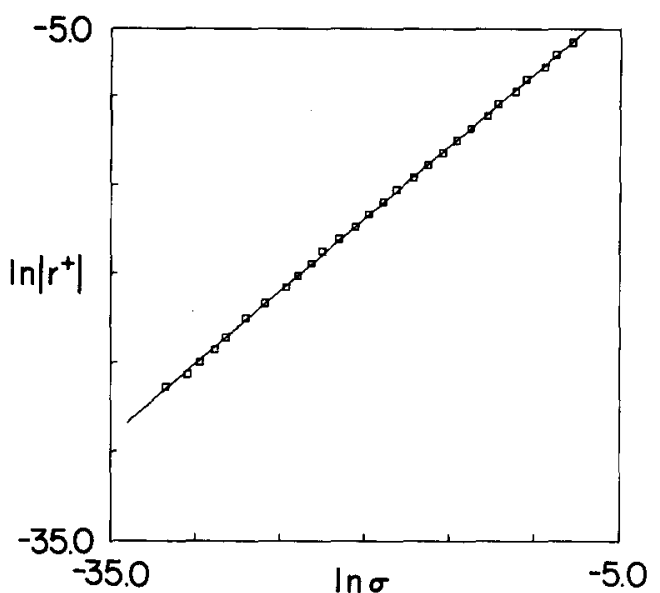

Fig. 18. Lowering of the chaotic threshold $r^{+}$with increasing noise level $\sigma$ for the $2^{n}$ cascade on a log-log scale. The absolute value of $r^{+}$is used as $r^{+}<0$. The 10 orders of magnitude in $r^{+}$correspond to orbits of periods from $2^{4}$ to $2^{16}$, in the deterministic limit. The noise level ranges over 12 orders of magnitude. Each data point comes from a binary search at fixed $r$ of a $\lambda$ vs. $\sigma$ curve for the zero-crossing of $\lambda$. During the search each $\lambda$ was calculated with $N=10^{6}$ or $10^{7}$ (small $\sigma$ ) in eq. (2-11).

for both $q=1$ and 3 , and

$$
k_{1}=0.60 \pm 0.01, \quad k_{3}=0.40 \pm 0.01 \text {. }
$$

Figure 18 shows a $\log -\log$ plot of $r^{+}$versus $\sigma$ over 12 orders of magnitude in $\sigma$. The range in $r$ corresponds, in the deterministic limit, to orbits from period $2^{4}$ to period $2^{16}$.

If we recall the definition of $\sigma_{p}$ as the noise level at which one can observe an orbit of at most period $p$ and if we define $r^{+}\left(\sigma_{p}\right)=r_{p}^{+}$, then eq. (2-9) written in terms of the normalized parameter gives

$$
\bar{r}_{p} / \bar{r}_{2 p}=\delta
$$

and, also,

$$
r_{p}^{+} / r_{2 p}^{+}=\delta
$$

Using eq. (4-1), we then obtain [1]

$$
\sigma_{p} / \sigma_{2 p}=\delta^{1 / \gamma}
$$

Thus for a cascade where one can observe at most a period of $p$ at a given noise level $\sigma_{p}$, one must reduce the noise level by a factor of $\delta^{1 / \gamma} \sim 6.6$ in order to resolve a period of $2 p$.

The behavior of $\lambda$ near $r_{c}$ in the absence and presence of noise as shown in figures 9 and 17, leads to the notion of external fluctuations acting as a disordering field on the deterministic dynamics. As we pointed out in section 2 , in the deterministic limit $\lambda$ behaves as a disorder parameter for chaos, with a power law given by eq. (2-12). As the strength of the external noise is increased, $\lambda$ acquires a finite value 


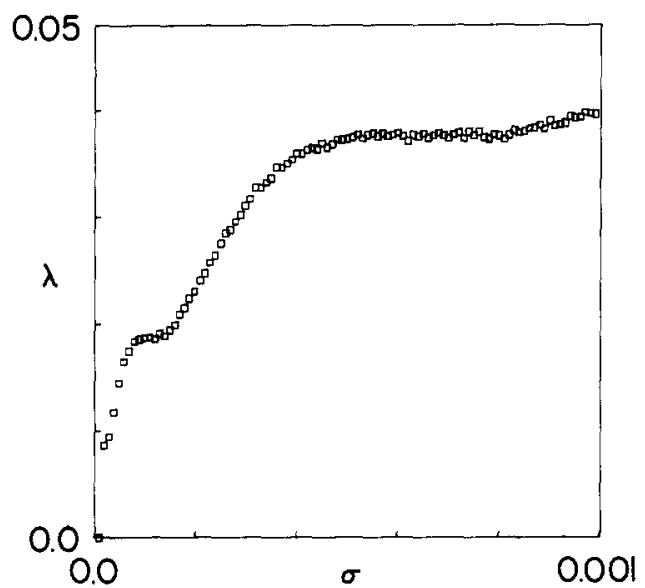

Fig. 19. Characteristic exponent $\lambda$ versus noise level $\sigma$ at $r_{c}$ for the $2^{n}$ cascade. $\lambda$ was calculated with $N=10^{6}$ in eq. (2-11) for 100 values of $\sigma$. The steps correspond to the noise-induced merging of bands. The overall power law behavior, though, can be summarized by the critical exponent $\theta$ of eq. (4-7).

at $r_{c}$, while the zero crossing is renormalized according to eq. (4-1). Furthermore, since the presence of a bifurcation gap implies the smearing of bands in the chaotic regime, $\lambda$ will no longer possess an infinite slope at the threshold in the presence of a disordering field.

These considerations lead us naturally to expect that at $r_{c}$, the Lyapunov characteristic exponent $\lambda$ will scale with noise according to

$$
\lambda(r, s)=A \sigma^{\theta}
$$

where $\theta$ is a universal exponent which plays a role similar to the isothermal exponent in critical phenomena [34]. Figure 19 shows the dependence of the characteristic exponent at $r=r_{\mathrm{c}}$ on the noise level $\sigma$. In addition to an overall power law behavior, several "steps" are apparent in the curve of $\lambda(\sigma)$. The steps are separated by factors of $\delta^{1 / \gamma} \sim 6.6$ in the noise level and correspond to the noise-induced merging of bands. The sequence of probability distributions of fig. 20 illustrates the rescaling of the dynamics by changes in the noise level of factors of $\delta^{1 / \gamma}$. As the noise increases by $\delta^{1 / \gamma}$ from (a) to (b) and again from (b) to (c), the structure of the band probability distribution is seen to be the same, except for a spatial rescaling. Figure 19 also shows that $\lambda$ increases relatively linearly with noise level until bands merge, at which point $\lambda$ levels off with increasing noise level because the probability density changes little. This is verified by the noise bifurcation diagram of fig. 21 which shows 16 bands merging into 8 and 8 bands merging into 4 at noise levels corresponding to the steps in $\lambda(\sigma)$ of fig. 19 .

In order to determine the validity of eq. (4-7) we have measured the change of $\lambda$ at $r=r_{\mathrm{c}}$ with increasing noise level over 9 orders of magnitude in $\sigma$. As can be seen in fig. 22, a log-log plot of the data reveals a straight line from which we can extract the values

$$
\theta=0.37 \pm 0.01, \quad A_{1}=0.58 \pm 0.01
$$

for the period 1 cascade and

$$
\theta=0.37 \pm 0.01, \quad A_{3}=1.13 \pm 0.01
$$



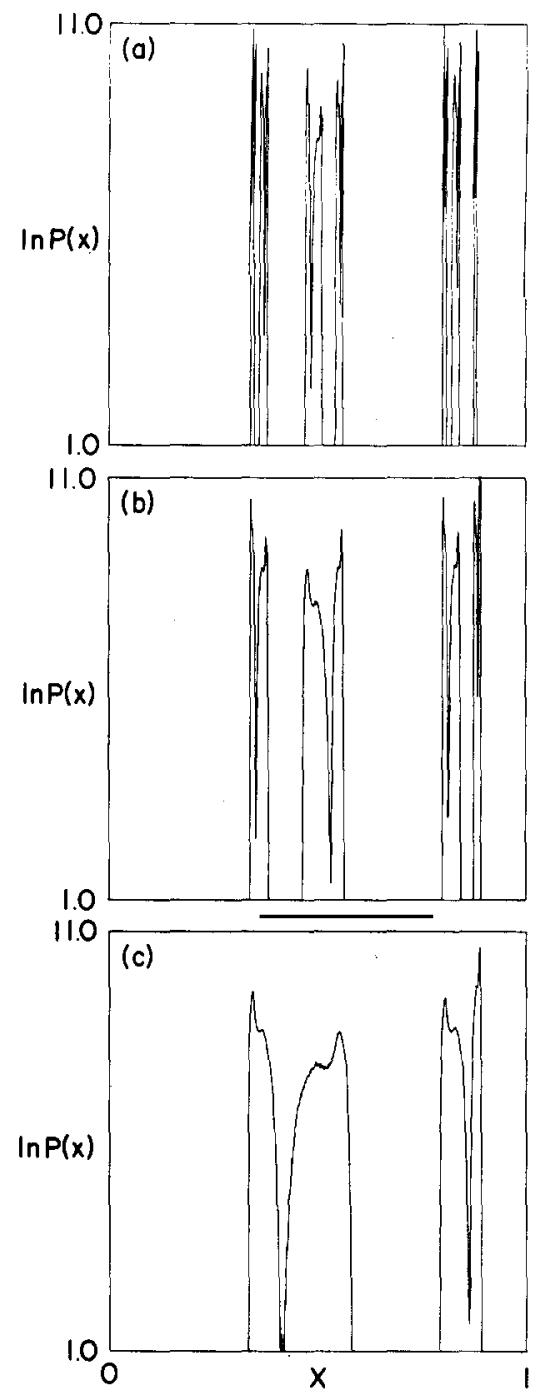

Fig. 20. Logarithmic plot of (unnormalized) probability density at the accumulation point $r_{c}=3.569945 \ldots$ of the $2^{n}$ cascade for three different noise levels separated by factors of $\delta^{1 / y} \sim 6.6$ : (a) $\sigma=2.3 \times 10^{-5}$, (b) $\sigma=1.5 \times 10^{-4}$, and (c) $\sigma=10^{-3}$. The last corresponds to the noise level of fig. 7 in which 4 bands are apparent. The sequence illustrates the band-merging bifurcations at $r_{\mathrm{c}}$ induced by increasing noise levels. Again, $10^{6}$ iterations of eq. (3-1) were partitioned into $10^{3}$ bins. The difference in noise levels is chosen to illustrate the noise scaling structure of $P(x)$.

for the period 3 cascade. The deviations from a straight line, seen as groupings of 2 and 3 data points in fig. 22, are due to the noise-induced bands mergings found in fig. 19.

These results together with the Huberman-Rudnick scaling of the characteristic exponent, eq. (2-12), and recent scaling theories $[35,36]$, suggest the existence of a homogeneous scaling function $F[\bar{r}, \sigma]$ such that

$$
\lambda(\bar{r}, \sigma)=-\frac{\mathrm{d}}{\mathrm{d} \sigma} F[\bar{r}, \sigma]
$$




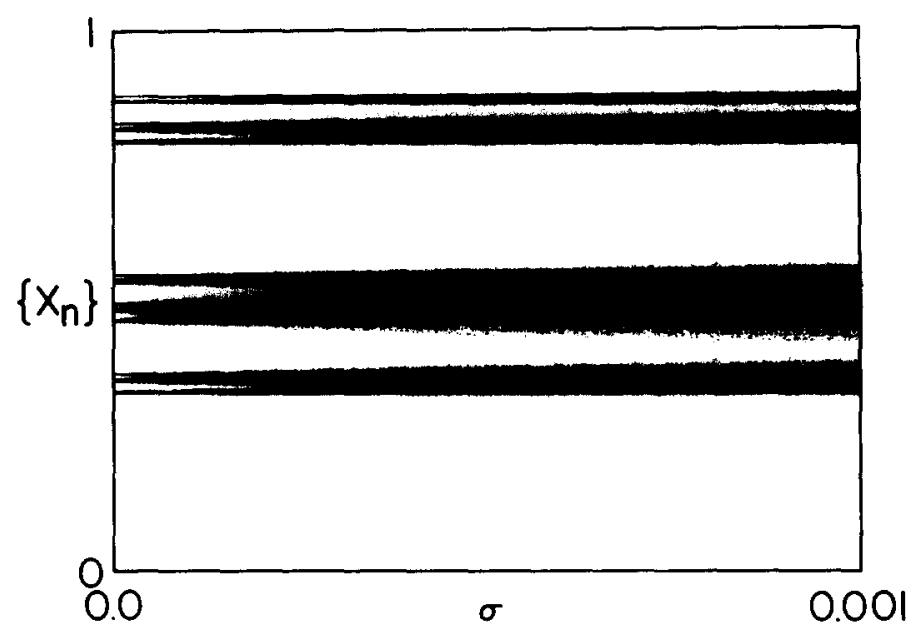

Fig. 21. Noise bifurcation diagram diagram at $2^{n}$ accumulation point: attractor $\left\{x_{n}\right\}$ versus noise level $\sigma$ at $r_{\mathrm{c}}=3.56995 \ldots .500$ iterates of eq. (3-1) are plotted after an initial 500 iterations at each of the $10^{3}$ increments in $\sigma$.

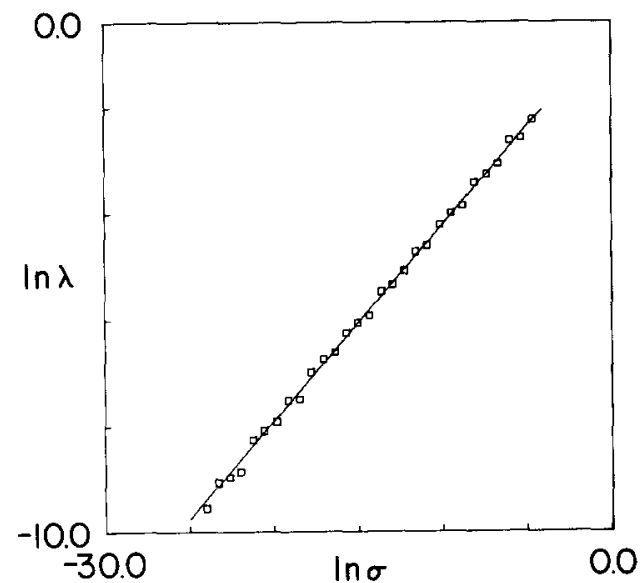

Fig. 22. $\log -\log$ plot of $\lambda$ versus noise level $\sigma$ showing the data used to obtain $\theta=0.37$ and $A_{1}=0.58$ for eq. (4-7). The groupings of 2 and 3 data points reflect the noise-induced band mergings. $\lambda$ was calculated with $N=10^{6}$ in eq. (2-11) at 30 values of $\sigma$ which ranged over 9 orders of magnitude.

and satisfying a scaling behavior, i.e.

$$
F\left[L^{a_{r}} \bar{r}, L^{a_{\sigma}} \sigma\right]=L F[\bar{r}, \sigma],
$$

where $a_{r}$ and $a_{\sigma}$ are scaling parameters.

In particular, when $\sigma=0$, eqs. (4-9) and (4-10) imply

$$
\lambda(\bar{r}, 0)=(-\bar{r})^{\left(1-a_{\sigma}\right) / a_{r}} \lambda(-1,0)
$$

which together with eq. (2-12) gives

$$
\tau=\left(1-a_{\sigma}\right) / a_{r}
$$


Similarly, we can express the exponent $\theta$ in terms of the scaling parameters $a_{r}$ and $a_{\sigma}$. Setting $r=0$ in eqs. (4-9) and (4-10) and letting $\sigma \rightarrow 0$, we obtain

$$
\lambda(0, \sigma)=\sigma^{\left(1-a_{\sigma}\right) / a_{\sigma}} \lambda(0,1)
$$

which, together with eq. (4-7) gives

$$
\theta=\left(1-a_{\sigma}\right) / a_{\sigma}
$$

In order to obtain an explicit relation between $\tau$ and $\theta$, we can introduce a noise susceptibility $X(\bar{r}, \sigma)$ defined as

$$
X(\bar{r}, \sigma)=\mathrm{d} \lambda / \mathrm{d} \sigma
$$

The magnitude of the noise susceptibility $X$ gives a measure of the sensitivity of the dynamics to the addition of noise. For example, at the transitions to chaos and at points of superstability, $X$ diverges, although nearby it is finite and positive. Actually, near the transition to chaos, the behavior of $X$ is complicated somewhat by the accumulation of self-similar structure. The addition of a small amount of noise, however, truncates this structure so that the nature of the divergence at the transition can be studied in practice. Near $r_{\mathfrak{c}}$, then, $X$ should also obey a scaling law

$$
X(\bar{r}, 0)=c \bar{r}^{-\omega}
$$

Taking derivatives of eqs. (4-9) and (4-10) we obtain

$$
\omega=\left(2 a_{\sigma}-1\right) / a_{r}
$$

which together with eqs. (4-12) and (4-14) lead to the relation

$$
\omega=\tau\left(\theta^{-1}-1\right)
$$

Using the known values for $\tau$ and $\theta$, eq. (4-18) yields a prediction for the noise susceptibility critical exponent of $\omega=0.77$. In the previous section we noted that the addition of noise lowered the characteristic exponent at the period-doubling bifurcations. Thus $X$ is negative near period-doubling bifurcations and apparently diverges at this point of bifurcation too. We also pointed out that for a single chaotic band the characteristic exponent is affected very little over a wide range of noise level. In such cases, the noise susceptibility will be very small, if not zero.

The results of this section show that the existence of a homogeneous scaling function $F$ with universal properties leads to scaling relations very similar to those encountered in critical phenomena. In particular, one is able to accurately predict the value of the exponents that relate the effect of external fluctuations on the chaotic behavior of deterministic systems exhibiting period-doubling cascade bifurcations. Recent work $[35,36]$ points to a renormalization group description of the scaling behavior revealed by the above numerical investigations. In fact, these approaches yield critical exponents in excellent agreement with those reported above. 


\section{Concluding remarks}

Using the simplest dynamical system which undergoes cascade bifurcations we studied in a quantitative manner the fluctuation effects reported earlier in a system of nonlinear ordinary differential equations [1]. In particular, we have described the fluctuation-induced gap in the cascade bifurcation sequence and the scaling behavior of both the threshold noise and the disordering field. Our investigations provide strong evidence for the consideration of the Lyapunov characteristic exponent as a disorder parameter for chaos. We have also derived the scaling behavior of the effects of fluctuations using results from the universal scaling theory for the period-doubling bifurcation. Thus these results should be relevant to other dynamical systems, including weakly turbulent fluid flows, which undergo cascade bifurcation.

The existence of deterministic models that show chaotic or unpredictable behavior puts in a new light questions on the physical origins of noise processes. With this in mind, we would like to consider the larger context in which this paper should be placed. For models of classical physical systems we can distinguish three types of fluctuation. The first, observational noise, is due to the finite resolution of physical measurements, that is, instrumentation error. Problems associated with observational noise are often considered the province of the mathematical theory of communication, which describes the effect of random errors on a signal representing some physical quantity [37]. When a physical system is in contact with a "heat" bath in which a large number of particles (or degrees of freedom) are excited, a second type of fluctuation, external noise, appears. This second type of noise adds a stochastic force to the dynamical equations and the resulting non-deterministic problem is solved with statistical assumptions and techniques [38]. The Langevin equation describing Brownian motion exemplifies this class of stochastic model. Finally, chaotic dynamical systems exhibit stochasticity or random behavior, although they are completely deterministic. This deterministic randomness is the third type of fluctuation, which we shall call intrinsic noise. It is of interest, for example, to the study of nonlinear differential equations whose phase space descriptions require at least three dimensions.

In current attempts to relate deterministic chaotic models to turbulent physical systems, one of the outstanding problems is the interaction between each of these types of fluctuation. On one hand, noise appears as an everpresent but undesirable artifact which, more often than not, complicates experimentalists' interpretation of their data. On the other hand, from the theoretical point of view, the introduction of fluctuations in a problem implies a simplification of a model. Such an ansatz represents an explicit lower bound on the level of description below which the detailed dynamics are not to be considered. The results we have presented here and previously [1] suggest a role for fluctuations intermediate between the extremes of experimental complexity and theoretical simplicity. We have shown that a broad class of transitions to chaotic behavior can be characterized by its alteration in the presence of thermal-like noise. In principle, this should aid in distinguishing the type of model appropriate to describe observed, random behavior.

Aside from the particular behavior discussed here for the cascade bifurcation, we wish to emphasize the importance of considering fluctuations in modeling turbulent physical systems. From an understanding of the changes induced by fluctuations in the geometry of the phase space flow, one may be able to elucidate the relationship between simple chaotic dynamics and turbulent physical systems, such as turbulent fluid flow [39] and noisy solid state systems [1,5]. Currently, simple chaotic models serve only as metaphors for turbulent behavior in continuous (infinite dimensional) physical systems. Although one finds electronic and mechanical systems corresponding to chaotic models [40] and recent application of phase space reconstruction techniques to stirred chemical reactions [41], there 
is as yet no direct experimental evidence that chaotic dynamics describes observed random behavior in continuum systems, such as turbulent fluid flow. At present, the comparison of bifurcation sequences of power spectra between experiment and model systems and the reconstruction of phase space pictures provide the only methods of validation of the conjecture. Hopefully, an understanding of the relationship between the three types of fluctuation (observational, external and intrinsic) in model systems will lead to experimental tests which will determine the relevance of chaotic dynamics to noisy physical processes.

We should mention another approach to distinguishing between types of intrinsic noise that is of interest in distinguishing between intrinsic and thermal noise. It considers, first of all, the reconstruction of phase space dynamics from a single experimental variable and then determines the number of independent variables underlying the reconstructed dynamics, that is the intrinsic dimensionality of a phase space description [42,43]. According to this approach, different types of intrinsic noise require different numbers of phase space dimensions. Thermal noise, considered as a deterministic process, would be characterized by a relatively large number of phase space dimensions. It is still an open question whether practical algorithms can be devised to distinguish between thermal and intrinsic noise when the dimension of the underlying physical process is inherently large. At the present time, there appear to be substantial computational difficulties for the experimental determination of the intrinsic dimension of physical processes even when the dimension is as low as 5 (say), not to mention the problems of visualizing a chaotic attractor of that dimension with the reconstruction techniques currently proposed. The utility of these techniques will probably be limited in answering questions about the interaction of thermal noise and chaotic dynamics. Nonetheless, the conceptual framework that these techniques provide allows one to understand the transition from low dimensional chaotic dynamics to high dimensional, deterministic, thermal-like noise processes.

From a different perspective, Ruelle [44] and Shaw [19] estimate that the time necessary for a thermal fluctuation to affect the macroscopic motion of fully-developed turbulence in a fluid is relatively short, being on the order of seconds for air. Ruelle concludes, however, that in this regime, because thermal fluctuations must compete with many other perturbations of similar energy which are also amplified by the flow, changes in the level of thermal fluctuations would probably not be experimentally observable. In the weakly turbulent regime though, the changes in the level of thermal fluctuations could be quite noticeable, as suggested by our results on the cascade bifurcation to chaos. Fluctuations are of interest from a mathematical point of view, thermal fluctuations may also play an important role in selecting the relevant stationary measure on the chaotic attractor describing turbulence. In principle, there are many such measures, but, as Kifer [45] has shown for Axiom-A systems, only one is stable under small stochastic perturbations.

The picture of microscopic fluctuations determining macroscopic behavior is one that is generally associated with locally unstable or chaotic dynamics. Shaw [19] develops this notion by considering chaotic dynamical systems as sources of information; this information originates in the microscales beyond experimental resolution. He discusses the unpredictability of chaotic systems in terms of finite measurement resolution, or observational noise, to use the above terminology. As an example, he estimates how long it takes a chaotic system to become unpredictable. If the state of a system can be determined to within some finite resolution $\sigma$ (measured relative to the total number of resolvable states) and if one knows a priori the information loss rate $\lambda_{0}$ (such as the maximum Lyapunov characteristic exponent), then the system is effectively unpredictable a time $t=\ln (\sigma) / \lambda_{0}$ after a measurement. As a first approximation to an observation theory of chaos, this argument raises an interesting question for experimentalists: Without knowing the rate of information loss, how can the 
rate itself be measured experimentally in the presence of observational noise? Or, indeed, in the presence of the other types of noise mentioned above? [46].

As an explicit example of the relationship between thermal-like fluctuations and intrinsic noise, we have studied a model exhibiting chaotic behavior, eq. (3-1), which included a stochastic force. This "thermal fluctuation" term was in practice a deterministic pseudo-random number generator implemented on a digital computer. Its iterative algorithm was operated on a different time scale than the 1D map in order to let the correlations die out and so give the desired statistics. This time scale is determined by the degree of randomness of the algorithm which can be measured with a characteristic exponent, that is, a measure of the divergence of nearby states. The introduction of pseudo-random noise imposed a scale of resolution below which we did not consider the dynamics. This "thermal" noise was characterized by suitable statistical quantities, such as the mean and standard deviation, although it was a deterministic process. From the opposite perspective, this suggests that in pursuing the understanding of a noisy physical process to finer degrees of resolution, the "noise source" may appear as a deterministic nonlinear system with chaotic dynamics. This certainly was the case for our numerical experiments.

\section{Acknowledgements}

The authors have benefited from discussions with John Guckenheimer, Michael Nauenberg, Norman Packard, Joe Rudnick and Rob Shaw. They also wish to thank Ralph Abraham and Joe Rudnick for their support of the authors' computational work at UCSC and to acknowledge the help provided by the late Joe Maleson with the computers at Xerox PARC. Rob Shaw has generously provided fig. 2 from his unpublished work. This work was supported in part by the National Science Foundation Grant No. 443150-21299. JDF gratefully acknowledges the support of the Fannie and John Hertz Foundation. JPC was supported by a University of California Regents Fellowship.

\section{Appendix A. Fluctuations in a driven anharmonic oscillator}

Although characteristic exponents provide an unambiguous criterion for the early transition to chaos induced by increasing noise levels, for systems more complex than $1 \mathrm{D}$ maps, their calculation becomes quite time consuming. In the case of the driven anharmonic oscillator initially studied [1] one can observe this effect in the variation of Poincaré sections with noise level more readily, in fact, than in changes in the 1D map attractors with noise level. Generally, chaotic behavior appears with a particular degree of mapping, or folding, of orbits onto each other. Calculation of characteristic exponents gives the best determination of when this folding occurs in 1D maps. For driven oscillators, and other chaotic systems whose attractors appear sheet-like (of topological dimension two), on the other hand, this point is reached when the first folding appears in the attractor's geometry, as revealed in a sequence of Poincaré sections. From the underlying geometry of the attractor one can then infer the transition to chaos.

We include here two sets of Poincaré sections (figures A1 and A2), taken at different noise levels for the oscillator of ref. [1], to illustrate in another context the qualitative effects of fluctuations discussed in this paper. The anharmonic oscillator studied there is given by

$$
\ddot{x}+g \dot{x}+a x-b x^{3}=F \cos (w t) .
$$




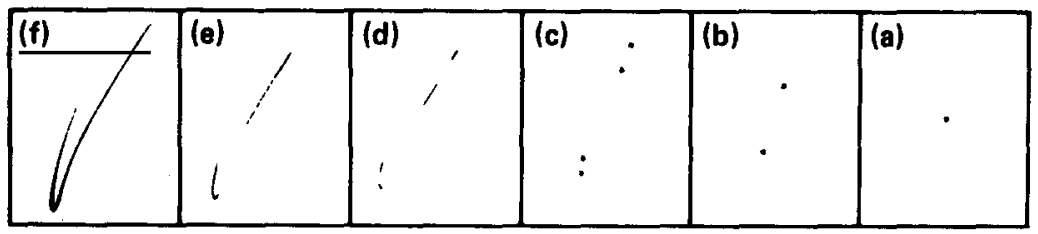

Fig. A1. A set of phase-zero Poincaré sections at different driving frequencies along the horizontal axis for the anharmonic oscillator studied in ref. [1] for a (nornalized) noise level $\sigma=10^{-3}$. The set (a)-(f) shows a cascade bifurcation as the driving frequency is lowered (toward the left in the figure). At this noise level only a maximum period of four can be observed. The folding geometry is apparent for the band attractors in (d)-(f).

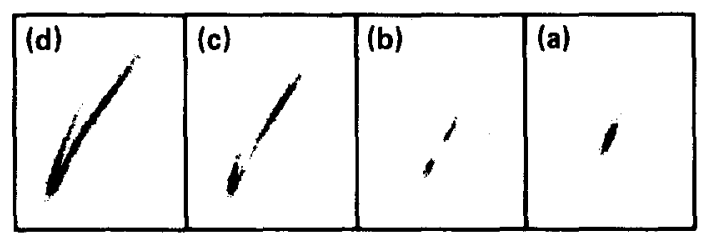

Fig. A2. The set of Poincaré sections (a)-(d) are similar to the first set in figure A1, except they are taken at a noise level $\sigma=10^{-2}$. Only a maximum period of two is present for this noise level. Notice also that the fluctuations spread more in the direction along the unstable manifold (bands) than along the stable manifold (transverse to the bands).

Figures 9 and 17 summarize the observable periods at a given noise level and periodicity, as is seen in figures $\mathrm{A} 1$ and $\mathrm{A} 2$. An important feature revealed by the Poincare sections is that the fluctuations spread out the orbits more along the "soft" directions within the attractor (the unstable manifold) than in the transverse directions (stable manifold), along which orbits contract very rapidly onto the attractor. The explanation for the induced transition to chaos with increasing noise level, then, is that the fluctuations fill out the folds of the attractor, causing the orbits to describe a geometry which is effectively chaotic. The existence of such folding geometry cannot be easily inferred from the bifurcation diagrams of the $1 \mathrm{D}$ map.

\section{Appendix B. Characteristic exponents in period-doubling regimes}

The characteristic exponent in periodic regimes, as seen in fig. 3, has a distinctive shape between period-doubling bifurcations. Although the dependence of the characteristic exponent $\lambda$ on the parameter $r$ cannot be analytically calculated in general, it can be straightforwardly determined from eq. (2-10) for a few simple cases. The nature of the divergence at superstable orbits can also be elucidated using eq. (2-10). Furthermore, once we have these results, a scaling of $\lambda(r)$ in the periodic regime, analogous to the scaling above $r_{\mathrm{c}}$ of eq. (2-12), follows easily.

A period $p$ orbit consists of the set of stable fixed points $\left\{x_{i}\right\}, i=1, \ldots, p$, of the $p$ th iterate of the map. These are given by the equation

$$
x_{i}=f^{p}\left(r, x_{i}\right)
$$

Eq. (B-1) implicitly determines the dependence of the fixed points $x_{i}$ on $r$. Thus, the bifurcation of periodic orbits reduces to the study of the real roots $x_{i}$ of the polynomial in $r, x_{i}-f^{p}\left(r, x_{i}\right)$. Only $p$ of these roots correspond to the points of the stable orbit; the others correspond to unstable orbits. For period 1 orbits of the logistic equation, we have the roots $x=0$ and 


$$
x_{1}=(r-1) / r
$$

The root $x_{1}$ describes the stable period 1 orbit for $r$ in $[1,3]$. For period 2, there are four roots of the polynomial

$$
x-f^{2}(r, x)=x-r^{2} x(1-x)(1-r x(1-x)) .
$$

The period 1 root and $x=0$ are also roots of this polynomial, but they correspond to unstable orbits. The remaining two roots describe the stable period 2 orbit for $r$ in $\left[3, r_{2-4}\right]$; they are

$$
x_{ \pm}=\frac{1+r \pm\left(r^{2}-2 r-3\right)^{1 / 2}}{2 r}
$$

Generally, a period $p$ orbit is determined by the roots of a polynomial of order $2^{p}$. For a period $2^{n}$ orbit, the roots corresponding to the unstable orbits of periods $2^{n-1}, 2^{n-2}, \ldots$, and 1 , can be factored out of the polynomial $x-f^{n^{n}}(r, x)$, in principle. This would leave a $2^{n}$-order polynomial whose roots are the points of the stable $2^{n}$ orbit.

From the equations for the dependence of the periodic orbits on $r$, the dependence of the characteristic exponent can be obtained from eq. (2-10). For a period $p$ orbit $\left\{x_{i}\right\}$, the probability density $P(x)$ is a set of $p$ delta functions, $\delta\left(x-x_{i}\right)$. In this case, eq. (2-10) becomes

$$
\lambda(r)=\sum_{i=1}^{p} \ln \left|f^{\prime}\left(r, x_{i}\right)\right| .
$$

For the logistic equation we have studied, the slope is given by

$$
f^{\prime}\left(r, x_{i}\right)=r\left(1-2 x_{i}\right)
$$

For the period 1 orbit, then, the characteristic exponent is

$$
\lambda(r)=\ln |2-r|
$$

for $r$ in $[1,3]$. The argument of the logarithm indicates that the bifurcations, where $\lambda \rightarrow 0$, from $x=0$ to the period 1 occurs at $r=1$ and from period 1 to period 2 occurs at $r=3$. It also shows that the superstable orbit, where $\lambda \rightarrow-\infty$, is found at $r=2$. Similarly for the period 2 orbit, we find

$$
\lambda(r)=\ln \left|r^{2}-2 r-4\right|
$$

for $r$ in $\left[3, r_{2-4}\right]$. The argument of the logarithm yields $r_{2-4}=1+\sqrt{6}$ and for the superstable period 2 orbit $r=1+\sqrt{5}$.

The characteristic exponent diverges at superstable orbits $\left\{x_{i}\right\}$ as one of the $x_{i}$ approach the critical point $x_{c}=0.5$, where the slope vanishes. To show this in general, we must first determine how the particular $x_{i}$ in question, denoted by $x^{*}$, approaches $x_{\mathrm{c}}$ as the parameter approaches the superstable value $r_{\mathrm{s}} . x^{*}$ is given by one branch of roots of the polynomial $x-f^{p}(r, x)$. This gives $x^{*}$ implicitly as a function of $r$; that is, if $g(r)$ is the single branch containing $x_{c}$, then we write 


$$
x^{*}(r)=g(r)
$$

where $x_{\mathrm{c}}=g\left(r_{\mathrm{s}}\right)$. As can be seen from the bifurcation diagram of fig. $1, g(r)$ is a smooth curve near $r_{\mathrm{s}}$. If we define $\mathrm{d} r=r-r_{\mathrm{s}}$, then

$$
\mathrm{d} x^{*}(r)=\frac{\mathrm{d}}{\mathrm{d} r} g(r) \mathrm{d} r
$$

Close to $r_{\mathrm{s}}$, the slope of $g(r)$ is very nearly a constant $k=(\mathrm{d} / \mathrm{d} r) g\left(r_{\mathrm{s}}\right)$, which gives

$$
\mathrm{d} x^{*} \sim k \mathrm{~d} r .
$$

Next, we must determine how the slope changes near $r_{\mathrm{s}}$. If we define $\mathrm{d} x=x^{*}-x_{\mathrm{c}}$ and $\mathrm{d} r$ as above, simple expansion gives

$$
f^{\prime}\left(r, x^{*}\right)=f^{\prime}\left(r_{\mathrm{s}}, x_{\mathrm{c}}+\mathrm{d} x\right) \sim f^{\prime}\left(r_{\mathrm{s}}, x_{\mathrm{c}}\right)+f^{\prime \prime}\left(r_{\mathrm{s}}, x_{\mathrm{c}}\right) \mathrm{d} x+\mathrm{O}\left(\mathrm{d}^{2} x\right) .
$$

By definition the first, third and higher, terms vanish. Furthermore, $f^{\prime \prime}(r, x)=-2 r$ so that we have

$$
f^{\prime}\left(r, x^{*}\right)=-2 r_{\mathrm{s}} \mathrm{d} x
$$

near $r_{\mathrm{s}}$. From eqs. (B-11) and (B-13), the slope's dependence on $r$ near $r_{\mathrm{s}}$ is then given by

$$
f^{\prime}\left(r, x^{*}\right) \sim-2 r_{\mathrm{s}} k \mathrm{~d} r
$$

The divergence of $\lambda(r)$ is dominated by the term in eq. (B-5) whose slope is vanishing, and so we can ignore the contributions from the other $(p-1)$ points along the orbit. Thus, we see from eq. (B-5) that the divergence of $\lambda(r)$ is logarithmic near $r_{\mathrm{s}}$ and given by

$$
\lambda(r) \sim \ln \left|2 r_{\mathrm{s}} k \mathrm{~d} r\right| \sim \ln \left|r_{\mathrm{s}}-r\right| .
$$

We now turn to the scaling properties of the superstable dips. As $r$ approaches $r_{c}$ the width $w_{n}$ of the $n$th dip in $\lambda(r)$ decreases exponentially at a rate given by

$$
w_{n}=r_{n+1}-r_{n} \sim \delta^{-n} .
$$

Furthermore, as the period doubles for each successive dip, the magnitude of $\lambda(r)$ decreases by a factor of two from its value on the previous dip. Within a single dip then, we can write

$$
\lambda_{n}(r)=\frac{\lambda_{0}}{2^{n}} \ln \left|\frac{2\left(r_{\mathrm{s}}-r\right)}{w_{n}}\right|
$$

where $\lambda_{0}$ is a constant and $r_{\mathrm{s}}$ is the value of the parameter $r$ at the superstable orbit. The depth of the superstable dips, as seen in fig. 3 , decreases as $r$ approaches $r_{c}$, although in principle, $\lambda(r)$ is infinite at the superstable orbits. This effect is due to the resolution in $r$ at which fig. 3 was made, but also reveals 
a scaling behavior of the superstable dips. This behavior can be accounted for by calculating the average of $\lambda(r)$ between period-doubling bifurcations. This average is given by

$$
\lambda_{n}=\frac{1}{w_{n}} \int_{r_{n}}^{r_{n+1}} \frac{\lambda_{0}}{2^{n}} \ln \left|\frac{2\left(r_{\mathrm{s}}-r\right)}{w_{n}}\right| \mathrm{d} r .
$$

Since the dips are nearly symmetric, the lower limit can be changed from $r_{n}$ to $r_{\mathrm{s}}$. Performing the integration we find

$$
\lambda_{n}=-\lambda_{0} / 2^{n}
$$

Solving eq. (2-9) for $n$ in terms of $r$, we find

$$
\lambda(r)=-\lambda_{0}^{\prime}\left(r-r_{\mathrm{c}}\right)^{\tau}
$$

where $\tau=\ln (2) / \ln (\delta)=0.4498 \ldots$, and $\lambda_{0}^{\prime}$ is a constant. Thus, we see that the average value of $\lambda(r)$ scales in a manner analogous to the envelope of positive characteristic exponent above $r_{\mathrm{c}}$, as shown in ref. [22].

Admittedly these are simple considerations. A more detailed analysis along these lines, as developed by Daido [47], shows that the argument of the logarithm in eq. (B-17) approaches a universal polynomial. Eqs. (B-7) and (B-8) are the first approximations to the universal expression for the Lyapunov exponent in the period doubling regime.

\section{Appendix C. Equivalence of parametric and additive noise}

The equivalent parametric noise rule, introduced in section 3, allows one to construct a good approximation to the noisy bifurcation diagram of fig. 7 from the deterministic bifurcation diagram of fig. 1. Simply stated, the noisy bifurcation diagram is obtained by the convolution of the deterministic bifurcation diagram with a Gaussian probability distribution in $r$ whose standard deviation is given by eq. (3-12b). A good approximation of this process can be constructed in the following manner: In a piece of paper cut a slit that is parallel to the $x$ axis of fig. 1. The width of the slit in the $r$ direction should be equal to several standard deviations of the equivalent parametric fluctuations. To estimate the noisy attractor at any particular value, place the slit over fig. 1 so that its midline lies at the parameter value of interest. Now project all the points of the neighboring attractors that are visible within the slit onto the midline. If the width of the slit is appropriately chosen, the resulting distribution of points will give the attractor in the presence of noise.

As a first estimate of the proper slit width, we assume that the slit width is constant in $x$ and use eq. (3-12b). If the slit width is set at $3 \sigma_{q}$, then $99.9 \%$ of the equivalent parametric fluctuations will have magnitudes that lie within the slit. For the example of fig. 7 the magnitude of the additive fluctuations is $\sigma_{p}=10^{-3}$ and the estimated slit width is $3 \sigma_{q}=24 \sigma_{p}=0.024$. That is, the slit width to be used with fig. 1 to obtain fig. 7 should be $2.4 \%$ of the horizontal axis.

Let us now construct an approximation of fig. 7 using such a slit and fig. 1. Notice that the slopes of the bifurcation curves in fig. 1 are large near the period-doubling bifurcation to period 4 . Consequently, the noisy bifurcation curves of fig. 7 are broadened near this point by the projection onto the midline of 
the slit. Similarly, the bifurcation to the period 8 orbit is so close to the period 16 that the values of $x$ visible within the slit never separate. The period 8 orbit, and all higher period orbits, never become visible in the noisy bifurcation diagram of fig. 7. The other noise effects discussed in section 3 can be explained in an analogous manner.

This approximation neglects the $x$-dependence of the equivalent parametric fluctuations. Notice, for example, that the width of the upper fork of the period 2 bifurcation curve is much thinner than that of the lower fork. One of the reasons for this is apparent from the slit construction: the upper bifurcation curve has a smaller slope. Another reason is that the equivalent fluctuations are not ergodic. Eq. (3-6) predicts that the $q_{n}$ fluctuations are larger when $x_{n} \sim 0.8$, i.e. the upper fork, than they are when $x_{n} \sim 0.5$, i.e. the lower fork. At first glance this may appear to have an effect counter to that which we are trying to explain. However, the influence of the larger fluctuations on the upper fork is only felt on the succeeding iteration, that is, by the lower fork. Both of these effects combine to make the upper branch for fig. 7 considerably narrower than the lower.

In order to take this second effect into account automatically, rather than using a slit of fixed width, the width can be varied as a function of $x$. To do this it is necessary to consider the amplitude of the parametric fluctuations as a function of the value of $x$ on the next iteration, when they are most strongly felt. From eq. (3-5) we see that

$$
p_{n}=q_{n} f\left(x_{n}\right)
$$

and that

$$
f\left(x_{n}\right)=\left(x_{n+1}-p_{n}\right) / r .
$$

Assuming $p_{n} \ll 1$ and eliminating $f\left(x_{n}\right)$ from eqs. (C-1) and (C-2), we find

$$
q_{n} \sim r p_{n} / x_{n+1} .
$$

The fluctuation $q_{n}$ is felt on the $(n+1)$ st iteration; that is, $q_{n}$ affects $x_{n+1}$. Thus, at any given value of $x$ the slit width $w(x)$ is given by

$$
w(x)=3 \sigma_{q}(x)=3 r \sigma_{p} / x,
$$

if one takes three standard deviations.

\section{References}

[1] J.P. Crutchfield and B.A. Huberman, Phys. Lett. 74A (1980) 407.

[2] B.A. Huberman and J.P. Crutchfield, Phys. Rev. Lett. 43 (1979) 1743.

[3] A. Libchaber and J. Maurer, J. de Physique 41 (1979) 51;

J.P. Gollub, S.V. Benson and J. Steinman, A Subharmonic Route to Turbulent Convection, in: Annals of the New York Academy of Sciences 357 (1980) 22.

[4] Yu.N. Belyaev, A.A. Monakhov, S.A. Scherbakov and I.M. Yavorshaya, JETP Lett. 29 (1979) 295.

[5] B.A. Huberman, J.P. Crutchfield and N.H. Packard, App. Phys. Lett. 37 (1980) 750.

[6] J. Crutchfield, D. Farmer, N. Packard, R. Shaw, G. Jones and R. Donnelly, Phys. Lett. 76A (1980) 1.

[7] E.N. Lorenz, Annals of the New York Academy of Sciences 357 (1980) 282; and J. Crutchfield, D. Farmer, R. Shaw, G. Jones and R. Donnelly, Phys. Lett. 76A (1980) 1.

[8] Y. Kuramoto found the cascade bifurcation in a set of nonlinear partial differential equations: Diffusion-Induced Chemicai Turbulence, Physics Department, Kyoto University, Japan, preprint (1979).

[9] M. Feigenbaum, J. Stat. Phys. 19 (1978) 25; 21 (1979) 669.

See also C. Tresser and P. Coullet, CR. Acad. Sci. 287A (1978) 577.

[10] P. Collet and J.-P. Eckmann, Iterated Maps of the Interval as Dynamical Systems, Birkhauser (1980), and references therein.

[11] Y. Oono and M. Osikawa, Prog. Theo. Phys. 64 (1980) 54. 
[12] For a pictorial introduction to dynamical systems theory see R.H. Abraham and C.D. Shaw, Dynamics: The Geometry of Behavior (Aerial Publications, Santa Cruz, California, 1982).

For a mathematical introduction to dynamical systems theory see D.R.J. Chillingworth, Differential Topology with a View to Applications (Pitman, 1976),

or J. Guckenheimer, J. Moser and S.E. Newhouse, Dynamical Systems (Birkhauser, 1980).

[13] E.N. Lorenz, J. Atmos. Sci. 20 (1963) 130.

[14] R. May, Nature 261 (1976) 459.

[15] Numerical investigations of the features discussed here were also reported by S. Grossmann and S. Thomae, Z. Naturforschung 32A (1977) 1353.

[16] B. Mandelbrot, Fractals: Form, Chance, and Dimension (W.H. Freeman, San Francisco, California, 1977).

[17] J. Milnor and W. Thurston, Institute for Advanced Study, Princeton University, preprint (1977).

[18] A.N. Kolmogorov, Dokl. Akad. Nauk. SSSR 119 (1958) 861.

[19] R. Shaw, Z. Naturforschung 36a (1981) 80.

[20] J. Guckenheimer, Commun. Math. Phys. 70 (1979) 133.

[21] For the general theory of Lyapunov characteristic exponents see V.I. Oseledec, Trans. Moscow Math. Soc. 19 (1968) 197 ; Ja.B. Pesin, Russ. Math. Surv. 32 (1977) 55.

[22] B.A. Huberman and J. Rudnick, Phys. Rev. Lett. 45 (1980) 154.

[23] J.P. Crutchfield and R. Shaw, unpublished; T. Geisel, J. Nierwetberg and J. Keller, Phys. Lett. 86A (1981) 75.

[24] At $r=4.0$ the map becomes fully two-onto-one, taking the entire interval onto itself with one fold. $\lambda$ can be calculated directly at this point from eq. (2-10) to find $\lambda(4.0)=\ln (2)$; in other words, one bit of information is lost per iteration.

[25] P. Grassberger, Physics Dept., University of Wupertal, Germany, preprint WU-B-80-33 (1980).

[26] B.A. Huberman and A. Zisook, Phys. Rev. Lett. 46 (1981) 626; J.D. Farmer, Phys. Rev. Lett. 42 (1981) 179;

S. Grassmann, J. Stat. Phys. 26 (1981) 485.

[27] G. Mayer-Kress and H. Haken, J. Stat. Phys. 26 (1981) 149.

[28] J. Yorke and E. Yorke, J. Stat. Phys. 21 (1979) 263.

[29] P. Manneville and Y. Pomeau, Phys. Lett. 75A (1979) 1.

[30] P. Manneville and Y. Pomeau, Commun. Math. Phys. 79 (1980) 189.

[31] J.P. Eckmann, L. Thomas and P. Wittmer, J. Phys. A 14 (1982) 3153.

[32] J.E. Hirsch, B.A. Huberman and D.J. Scalapino, Phys. Rev. A 25 (1982) 519.

[33] H. Haken and G. Mayer-Kress, Z. Phys. B 43 (1981) 185.

[34] S.-K. Ma, Modern Theory of Critical Phenomena (Benjamin, Massachusetts, 1976).

[35] B. Shraiman, C.E. Wayne and P.C. Martin, Phys. Rev. Lett. 46 (1981) 935.

[36] J.P. Crutchfield, M. Nauenberg and J. Rudnick, Phys. Rev. Lett. 46 (1981) 933.

[37] C.E. Shannon and W. Weaver, The Mathematical Theory of Communication (University of Illinois Press, 1962).

[38] S. Chandrasekhar, Rev. Mod. Phys. 15 (1943) 1.

[39] D. Ruelle and F. Takens, Comm. Math. Phys. 50 (1976) 69.

[40] See, for example, Y. Ueda, C. Hayashi and N. Akamatsu, Elect. Comm. Jap. 56-A (1973) 27;

P.J. Holmes, Phil. Trans. Roy. Soc. Lond. 292 (1979) 419;

F.C. Moon, Experimental Models for Strange Attractor Vibrations in Elastic Systems, in: New Approaches to Nonlinear Problems in Dynamics, ed. P.J. Holmes (S.I.A.M, Philadelphia, 1980).

[41] J.C. Roux, A. Rossi, S. Bachelart and C. Vidal, Phys. Lett. 77A (1980) 391.

[42] N.H. Packard, J.P. Crutchfield, D. Farmer and R. Shaw, Phys. Rev. Lett. 45 (1980) 712;

H. Froehling, J.P. Crutchfield, J.D. Farmer, N.H. Packard and R. Shaw, On Determining the Dimension of Chaotic Flows, Physica 3D (1981) 605.

[43] F. Takens, Detecting Strange Attractors in Turbulence, to appear in the Proceedings of the Warwick Symposium (1980).

[44] D. Ruelle, Phys. Lett. 72A (1979) 81.

[45] Ju.I. Kifer, USSR Izvestija 8 (1974) 1083.

[46] The following references represent one approach to these questions:

J.P. Crutchfield and N.H. Packard, Int'। J. Theo. Phys. 21 (1982) 433; also in: Evolution of Ordered and Chaotic Patterns in Systems Treated by the Natural Sciences and Mathematics, ed. H. Haken (Springer-Verlag, Berlin, 1982); and to appear in the Proceedings of the Order in Chaos Conference, Los Alamos, New Mexico (1982).

[47] H. Daido, Phys. Lett. 83A (1981) 246. 